\title{
Assessing The Relative Performance Of Heavy-Tailed Distributions: Empirical Evidence From The Johannesburg Stock Exchange
}

\author{
Chun-Sung Huang, University of Cape Town, South Africa \\ Chun-Kai Huang, University of Cape Town, South Africa \\ Knowledge Chinhamu, University of KwaZulu-Natal, South Africa
}

\begin{abstract}
It has been well documented that the empirical distribution of daily logarithmic returns from financial market variables is characterized by excess kurtosis and skewness. In order to capture such properties in financial data, heavy-tailed and asymmetric distributions are required to overcome shortfalls of the widely exhausted classical normality assumption. In the context of financial forecasting and risk management, the accuracy in modeling the underlying returns distribution plays a vital role. For example, risk management tools such as value-at-risk (VaR) are highly dependent on the underlying distributional assumption, with particular focus being placed at the extreme tails. Hence, identifying a distribution that best captures all aspects of the given financial data may provide vast advantages to both investors and risk managers. In this paper, we investigate major financial indices on the Johannesburg Stock Exchange (JSE) and fit their associated returns to classes of heavy tailed distributions. The relative adequacy and goodness-of-fit of these distributions are then assessed through the robustness of their respective VaR estimates. Our results indicate that the best model selection is not only variant across the indices, but also across different VaR levels and the dissimilar tails of return series.
\end{abstract}

Keywords: Heavy-Tailed and Asymmetric Distributions; Value-At-Risk (VaR); Johannesburg Stock Exchange

\section{INTRODUCTION}

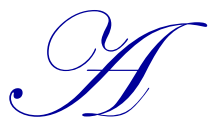

topic of ongoing research is the identification of the most suitable and accurate distribution to fit financial returns data. Accomplishing such novel finding may prove particularly useful in the context of financial forecasting and risk management. The modeling of financial returns distribution was classically reliant on the normality assumption. However, a wealth of studies has shown that financial time series exhibits substantial skewness and excess kurtosis that contradicts Gaussianity (Tsay, 2010). Alternative implementations, such as the generalized autoregressive conditional heteroscedastic model (GARCH) of Bollerslev (1986), the Student's $t$-distribution (Huisman et al., 1998) and other skew $t$ innovations (Azzalini \& Capitanio, 2003; Jones \& Faddy, 2003), have also been suggested by researchers in an attempt to overcome such contradictions.

Recently, it has been proposed that the classes of generalized extreme value distributions and generalized Pareto distributions (Diebold et al., 2000; Bali, 2003; Rocco, 2014) and the class of generalized hyperbolic distributions (Eberlein \& Keller, 1995; Eberlein \& Prause, 2002; Hu \& Kercheval, 2007) provide a more robust modeling of financial returns distribution. However, to the best of our knowledge, there has been limited study on the cross-comparison between the performance of these models and their related applications such as in the context of risk management. In particular, a gap exists in the current literature that determines which model may best forecast the rate of occurrence of extreme events and, as a result, yield the most precise value-at-risk (VaR) estimates for financial institutions to measure market risk and adjust for adequate capitalization as per the Basel Regulatory Framework. 
Furthermore, whether it is possible to identify a model that provides the most accurate VaR estimate over all significance levels is not immediately clear. In addition, investigations also need to be extended to evaluate the differences between model performances over both long and short positions of trade. In this paper, we conduct various statistical examinations to make robust conclusions on the above claims and the adequacy of the suggested models to fit financial data. In particular, we focus on the cross-comparison analyses to identify the relative performances between proposed heavy tailed distributions. Motivations were partially drawn from Vee et al. (2012) whom determined that the return series of different indices may be best depicted by different distributions.

In this paper, we analyze the major indices on the Johannesburg Stock Exchange (JSE). South Africa has the largest and most developed economy amongst the sub-Saharan African countries. It is considered a cornucopia of mineral resources, with a well capitalized banking structure, sound regulatory and oversight practices, as well as research and development capabilities, and has an established manufacturing foundation. The Johannesburg Stock Exchange (JSE) Top40 Index is also a member of the BRICS Exchanges Alliance. ${ }^{1}$ As a member of the G-20, South Africa's financial market development was ranked third out of 148 countries in the World Economic Forum's Global Competitiveness Report 2013-2014. Notably, it was ranked first within the financial market development pillar due to its regulation of securities exchanges and legal rights index. Due to these prudent fiscal and monetary policies, the South African capital markets were not as largely affected by the global financial crisis as its international counterparts. Consequently, South Africa remains an attractive low-risk destination for many investors worldwide, with a sophisticated market structure and one of the largest exchanges in developing countries. Not only would our analysis provide a better insight into the ability of heavy tailed distributions to capture the anomalies embedded in the returns data of South African market, but it will also provide a glimpse into a cross-comparison of the performance of these models in emerging markets. It is also worthwhile mentioning that the unique characteristics of emerging markets are dissimilar to those of developed markets. Better modeling of the financial returns within emerging markets continues to draw much attention from academics and practitioners worldwide.

We begin our investigation by fitting different heavy-tailed distributions to the various indices. In addition to the three classes of distributions mentioned above, we also include four other well-known heavy-tailed distributions in our analyses; namely, Burr XII (Burr, 1942; Singh \& Maddala, 1976), Johnson SU (Johnson, 1949), hyperbolic secant (Baten, 1934; Harkness \& Harkness, 1968), and Dagum (Dagum, 1975, 1977) distributions. The selection of these distributions is based on some of their attractive properties (such as, the ability to capture asymmetry, non-identical tail behaviors, excess kurtosis, and the depiction of both heavy and semi-heavy tails) that are particularly useful in capturing the various stylized facts embedded in financial data. Goodness-of-fit tests are first conducted before the performances of these models are assessed through across-comparison of their relative VaR estimations. We make use of the widely recognized Kupiec likelihood ratio test and the Christoffersen test to conduct our backtesting before drawing robust conclusions on our analyses of the VaR estimates.

Our primary objective is the attempt to identify the most adequate distributional assumption that may fully capture the unique characteristics and stylized facts exhibit by the returns data of different indices. Furthermore, we examine the adequacy and goodness-of-fit by investigating their corresponding VaR estimates. Surprisingly, our results show that the best model selection is not only variant across different indices, but also changes across different VaR levels and the dissimilar tails in the return series.

The remainder of the paper is structured as follows. Section 2 commences by giving a descriptive statistical summary of the indices and Section 3 introduces the various heavy-tailed distributions under study. Section 4 describes the well-known value-at-risk measure and the related backtesting procedures. Empirical results from fitting the distributions to the indices are provided and analyzed in Section 5. Finally, Section 6 concludes the article and comments on possible further research.

\section{DESCRIPTIVE ANALYSES OF DATA}

The data used in this research includes eight major indices on the JSE, which were supplied by McGregor BFA. They are part of the FTSE/JSE Africa Index Series formulated to represent the performance of companies and

${ }^{1}$ Which further comprises of Brazil's Bovespa Index, Russia's Micex Index, the BSE India Sensitive Index, Hong Kong's Hang Seng Index, and the Hang Seng China Enterprises Index.

Copyright by author(s); CC-BY 
different market sectors in South Africa. The daily closed value for JSE All Share Index (ALSI/J203), JSE Top 40 Index (ALSI40/J200), JSE Resource 10 Index (RESI10/J210), JSE RAFI All Share Index (RAFI/J263), JSE SA Financials and Industrials Index (FINDI/J250), and JSE Capped All Share Index (CALSI/J303) were recorded from 17 December 2003 to 17 December 2013, while the JSE All Africa 40 Index (Africa40/JA00) daily figures were recorded from 16 May 2011 to 17 December 2013 and the JSE South African Volatility Index (SAVI) values were recorded from 1 February 2007 to 17 December 2013.

The importance of the inclusion of such a variety of major indices in our investigation is two-fold. Firstly, their inclusion allows for a complete sweeping inference on the overall performance of the South African financial market. Furthermore, it provides ground for clearer scrutiny on the unique behaviors and characteristics of individual market sectors, as well as identifying which assumed heavy tailed distribution may best capture such properties. The All Shares Index (ALSI), for example, is identified and utilized as the benchmark index to measure the current performance of the South African market as a whole. It comprises roughly $99 \%$ of the total market capitalization on the JSE. The JSE Top 40 on the other hand comprises the largest 40 constituents of the ALSI on the basis of their market capitalization. Recognized as the large cap index, the JSE Top 40 accounts for more than $80 \%$ of the ALSI and is used as an alternative performance benchmark.

The RESI10 comprises of the top ten resources share on the JSE on the basis of market capitalization. Specifically, it is concentrated on the major mining companies in the South African market. In addition, Raubenheimer (2012) also indicated that more than $20 \%$ of the ALSI's weighting comprises of the two largest resource-mining companies. As a cornucopia of mineral resources, it becomes vital to understand the associated distinct characteristics within the mining sector. For example, given the susceptibility of the mining sector to various extreme events, such as the ever scrutinized mining sector strikes seen in the recent past, identifying a distribution that may improve the ability to capture such phenomena may provide an edge to risk managers and investors alike. The FINDI on the other hand, represents the financial and industrial sectors in South Africa. Finally, Raubenheimer (2012) also found a high level of concentration within the ALSI. The capped indices, such as the RAFI and CALSI, breaks away from the traditional price-based market capitalization weighting design system. In particular, the RAFI is derived based on the weighting of company fundamentals (e.g. sales, cash flow, book value and dividends). Analyzing market performance based on such methodology contributes a further dimension to the understanding of the current state of the market.

Log returns of the SAVI, considered as the "fear" gauge for the South African market, were also analyzed and fitted with the various heavy-tailed distributions. Apart from the sake of completeness, the inclusion of the SAVI in our cross-comparison may assist in further understanding of the volatility index in developing countries. The SAVI is commonly used as a tool to measure the market sentiment in South Africa's emerging market. Results from such analysis may draw interest from both academics and practitioners, and adds to the current body of knowledge regarding volatility indexes.

The return series for each index are calculated as the first backward-differences of the natural logarithm of the index values. For day $t$, the daily return $R_{t}$ is defined as:

$R_{t}=\ln \left(C_{t}\right)-\ln \left(C_{t-1}\right)$

where $C_{t}$ is the closed index value on day $t$.

Figure 1 presents the time series plot of the different index returns under consideration. The plots strongly indicate the presence of heteroscedasticity and volatility clustering in all return series, except for Africa40. The Africa40 return series exhibit a significantly lower volatility relative to other indices, while SAVI returns seem to have the highest volatility. Isolated extreme returns caused by shocks to the financial market may be noticed, such as the 2009 financial crisis (except for Africa40, where the data were only recorded post-crisis). Stationarity is also evidence from the plots, which is confirmed by utilizing the Augmented Dickey-Fuller (ADF) test and the Phillips-Perron (PP) unit root test given in Table 1. 

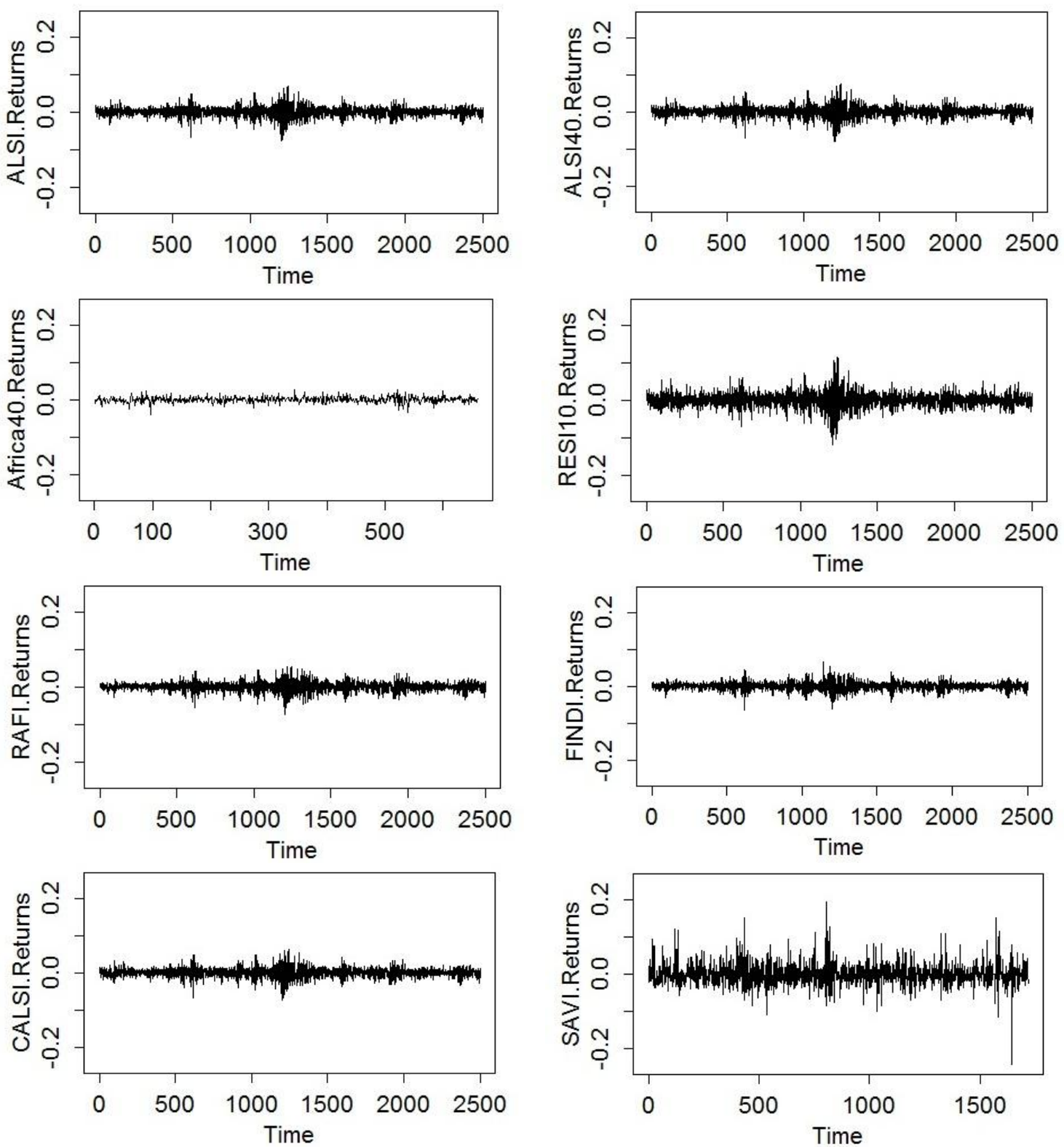

Figure 1: Time Series Plots for JSE Index Returns

The lag is set to zero for the ADF test using the Schwartz Information Criterion and the PP test is performed using the Newey-West estimator. For both tests, the $p$-values are interpolated from Banerjee et al. (1993). Results in Table 1 indicate that all return series are stationary by rejecting the null hypothesis of unit root.

Table 1: Results from ADF and PP Unit Root Tests of Stock Returns on Major JSE Indices

\begin{tabular}{|c|c|c|c|c|c|c|c|c|c|}
\hline & ALSI & ALSI40 & Africa40 & RESI10 & RAFI & FINDI & CALSI & SAVI \\
\hline \multirow{2}{*}{$\begin{array}{l}\text { ADF } \\
\text { test }\end{array}$} & Test statistic & -49.1197 & -49.7915 & -23.4318 & -47.9286 & -48.0545 & -49.0151 & -49.1079 & -41.5425 \\
\hline & $p$-value & $<0.01$ & $<0.01$ & $<0.01$ & $<0.01$ & $<0.01$ & $<0.01$ & $<0.01$ & $<0.01$ \\
\hline \multirow{2}{*}{$\begin{array}{l}\text { PP } \\
\text { test }\end{array}$} & Test statistic & -49.6797 & -50.6875 & -23.3435 & -48.3035 & -48.3252 & -49.2181 & -49.6585 & -41.5459 \\
\hline & $p$-value & $<0.01$ & $<0.01$ & $<0.01$ & $<0.01$ & $<0.01$ & $<0.01$ & $<0.01$ & $<0.01$ \\
\hline
\end{tabular}


Table 2: Descriptive Summary Statistics of Stock Returns on Major JSE Indices

\begin{tabular}{|l|c|c|c|c|c|c|c|c|}
\hline \multicolumn{1}{|c|}{ Index } & Minimum & Std. dev. & Skewness & Kurtosis & $\begin{array}{c}\text { Jarque-Bera } \\
\text { statistic }(\boldsymbol{p} \text {-value })\end{array}$ & Maximum & Mean & $\mathbf{N}$ \\
\hline ALSI & -0.0758068 & 0.012812 & -0.201463 & 6.681063 & $1428.964(<2.2 \mathrm{e}-16)$ & 0.0683397 & 0.0005942 & 2501 \\
\hline ALSI40 & -0.0795941 & 0.014021 & -0.13768 & 6.537586 & $1312.018(<2.2 \mathrm{e}-16)$ & 0.0770691 & 0.0005853 & 2501 \\
\hline Africa40 & -0.0407189 & 0.007743 & -0.182689 & 5.489705 & $173.8698(<2.2 \mathrm{e}-16)$ & 0.0280001 & 0.00066 & 659 \\
\hline RESI10 & -0.1181539 & 0.019071 & 0.002971 & 7.311042 & $1936.725(<2.2 \mathrm{e}-16)$ & 0.1149981 & 0.0003798 & 2501 \\
\hline RAFI & -0.0742110 & 0.011958 & -0.241772 & 5.891564 & $895.6661(<2.2 \mathrm{e}-16)$ & 0.0534232 & 0.0005752 & 2501 \\
\hline FINDI & -0.0647975 & 0.010741 & -0.227742 & 6.253189 & $1124.482(<2.2 \mathrm{e}-16)$ & 0.0652391 & 0.0006986 & 2501 \\
\hline CALSI & -0.0738210 & 0.012388 & -0.222274 & 6.536267 & $1323.738(<2.2 \mathrm{e}-16)$ & 0.0641731 & 0.0006096 & 2501 \\
\hline SAVI & -0.2434132 & 0.028942 & 0.503865 & 9.403844 & $3010.021(<2.2 \mathrm{e}-16)$ & 0.1944768 & -0.00005 & 1719 \\
\hline
\end{tabular}

A descriptive statistical summary of the different return series is provided in Table 2. Apart from SAVI returns, all other indices depict a positive mean. This indicates that the overall returns were slightly increasing over the period under investigation. All return series illustrate a small skewness, all negative except for RESI10 and SAVI. Such property is commonly found in financial series and relates to dissimilar tail behaviors in the data (Rydberg, 1999; Aas \& Haff, 2006). The high kurtosis' (all above 3) signifies leptokurtic behavior in these financial series, implying fatter tails in the actual distribution comparing to that of the Normal. This is further confirmed by the Jarque-Bera test, where the normality assumption is rejected for all indices.

The characteristics demonstrated in this section motivate the use of both symmetric and asymmetric heavy-tailed distributions for the modeling of these returns data and for the calculation of their corresponding VaR estimates.

\section{HEAVY-TAILED DISTRIBUTIONS AND PARAMETER ESTIMATION}

In this Section, we briefly introduce the various heavy-tailed distributions under consideration. In particular, we provide the probability density functions for hyperbolic, normal-inverse Gaussian, variance-gamma, generalized hyperbolic skew $t$, generalized extreme value, generalized Pareto, Burr XII, Johnson SU, hyperbolic secant, and Dagum distributions.

\subsection{The Class of Generalized Hyperbolic Distributions}

The generalized hyperbolic distributions (GHDs) were first introduced by Barndorff-Nielsen (1977) in an application to the mass-size distribution of aeolian sand deposits. The GHDs were later applied to financial data by other researchers, such as Eberlein and Keller (1995), Eberlein and Prause (2002), and Hu and Kercheval (2007). The family of GHDs portrays various beneficial properties for the modeling of financial data. For example, they cater for both skewness and symmetry; they are closed under conditioning, marginalization, and affine transformations; and, they allow for non-identical tail behaviors (Prause, 1999; Aas \& Haff, 2006).

We follow Prause (1999) for the parameterization of univariate generalized hyperbolic (GH) distribution. Suppose $X$ is a random variable following GHD, then its probability density function (pdf) can be defined as:

$f_{X}(x)=\frac{\left(\alpha^{2}-\beta^{2}\right)^{\lambda / 2} K_{\lambda-1 / 2}\left(\alpha \sqrt{\delta^{2}+(x-\mu)^{2}}\right) \exp (\beta(x-\mu))}{\sqrt{2 \pi} \alpha^{\lambda-1 / 2} \delta^{\lambda} K_{\lambda}\left(\delta \sqrt{\alpha^{2}-\beta^{2}}\right)\left(\sqrt{\delta^{2}+(x-\mu)^{2}}\right)^{1 / 2-\lambda}}$

where $K_{j}$ is the modified Bessel function of the third kind with order $j$ (Abramowitz \& Stegun, 1972) and the following conditions apply to the parameters:

$\begin{array}{ll}\delta \geq 0,|\beta|<\alpha, & \text { if } \lambda>0 \\ \delta>0,|\beta|<\alpha, & \text { if } \lambda=0 \\ \delta>0,|\beta| \leq \alpha, & \text { if } \lambda<0\end{array}$ 
We utilize the maximum likelihood estimation (MLE) for parameter estimates of the GHDs. Various subclasses of the GHDs are obtained via different assumptions made on the parameters. These special cases are given as below.

\subsubsection{Hyperbolic Distribution (Hyp)}

For $\lambda=1$, we get the hyperbolic distribution. The hyperbolic distribution is characterized by having a hyperbolic log-density function and exponential tails. Formally, a random variable has the hyperbolic distribution if its pdf is given by

$f_{H y p}(x)=\frac{\sqrt{\alpha^{2}-\beta^{2}}}{2 \alpha \delta K_{1}\left(\delta \sqrt{\alpha^{2}-\beta^{2}}\right)} e^{-\alpha \sqrt{\delta^{2}+(x-\mu)^{2}}+\beta(x-\mu)}$

where $K_{1}$ denotes the Bessel function of the third kind with index 1 . The first two of the four parameters, namely $\alpha$ and $\beta$, with $\alpha>0$ and $0 \leq|\beta|<\alpha$, determine the shape of the distribution with $\alpha$ representing the gradient and $\beta$, the skewness. $\delta>0$ is the scale parameter and $\mu \in \mathbb{R}$ is the location parameter.

\subsubsection{Normal-Inverse Gaussian Distribution (NIG)}

The normal-variance Gaussian distribution is a subclass of the GHDs with $\lambda=-1 / 2$. The pdf of NIG can be expressed as

$f_{N I G}(x)=\frac{\alpha \delta}{\pi} e^{\delta \sqrt{\alpha^{2}-\beta^{2}}+\beta(x-\mu)} \frac{K_{1}\left(\alpha \sqrt{\delta^{2}+(x-\mu)^{2}}\right)}{\sqrt{\delta^{2}+(x-\mu)^{2}}}$

where $K_{1}$ denotes the Bessel function of the third kind with index 1. The two tails of NIG are semi-heavy and non-identical. These make NIG attractive for financial applications (for example, see Anderson, 2001; Venter \& de Jongh, 2002). However, it is only appropriate when the two tails are not too heavy (Aas \& Haff, 2006).

\subsubsection{Variance-Gamma Distribution (VG)}

Setting $\lambda>0$ and let $\delta \rightarrow 0$ in Equation (1), we obtain the pdf of the variance-gamma distribution:

$f_{V G}(x)=\frac{\left(\alpha^{2}-\beta^{2}\right)^{\lambda}|x-\mu|^{\lambda-1 / 2} K_{\lambda-1 / 2}(\alpha|x-\mu|)}{\sqrt{\pi} \Gamma(\lambda)(2 \alpha)^{\lambda-1 / 2}} e^{\beta(x-\mu)}$

where $K_{\lambda-1 / 2}$ denotes the Bessel function of the third kind with index $\lambda-1 / 2$. The tails of VG decreases more slowly than the normal distribution, making it a suitable model for phenomena where extreme values are more probable than in the case of the normal distribution, such as returns from financial assets (Madan \& Senata, 1990).

\subsubsection{GH Skew t-Distribution (GHSt)}

Letting $\alpha \rightarrow|\beta|$ in Equation (1), we obtain the GH skew Student's $t$-distribution:

$f_{G H S t}(x)=\frac{2^{1 / 2+\lambda} \delta^{-2 \lambda}|\beta|^{1 / 2-\lambda} K_{1 / 2-\lambda}\left(\sqrt{\beta^{2}\left(\delta^{2}+(x-\mu)^{2}\right)}\right) \exp (\beta(x-\mu))}{\Gamma(-\lambda) \sqrt{\pi}\left(\sqrt{\delta^{2}+(x-\mu)^{2}}\right)^{1 / 2-\lambda}}$

where $\beta \neq 0$ and $\lambda<0$. If $\beta=0$, we get the non-central (scaled) Student's $t$-distribution. An important property of this distribution is that it has one heavy polynomial tail and one semi-heavy exponential tail. This makes it unique for modeling skewed data with dissimilar tail behaviors, such as commonly found in financial returns (Aas \& Haff, 2006). 


\subsection{Extreme Value Distributions}

The use of extreme value theory to model financial risk was first suggested by Diebold et al. (2000) and was followed by various work, such as Ho et al. (2000), Bali (2003), da Silva and de Melo Mendes (2003), Gençay and Selçuk (2004), and Gilli and Këllezi (2006). The main advantages of extreme value analysis are its ability to solely focus on the extreme observations (hence minimizing the bias caused by rest of the data), to cater for both asymmetry and heavy tails and to allow some extrapolation under certain conditions (Embrechts, 2000; Embrechts et al., 1999; McNeil \& Frey, 2000).

There are two general ways to identify extreme values in data, namely the block maxima method and the peaks-over-threshold approach. The former divides the data into blocks and selects the maximum observation in each block. The latter focuses on the realization of exceedances above a selected threshold (Coles, 2001). Two fundamental laws, the Fisher-Tippett-Gnedenko theorem (Fisher \& Tippett, 1928; Gnedenko, 1943) and the Pickands-Balkema-de Haan theorem (Pickands, 1975; Balkema \& de Haan, 1974), are associated with the two approaches, respectively, and give rise to the generalized extreme value distribution and the generalized Pareto distribution as limiting distributions. The asymptotic distribution of minima may be equivalently studied using the relation $\min \left\{X_{1}, \ldots \ldots, X_{n}\right\}=$ $\max \left\{-X_{1}, \ldots,-X_{n}\right\}$.

\subsubsection{Generalized Extreme Value Distribution (GEVD)}

The generalized extreme value distribution (GEVD) is used to model the maxima of a long, but finite, sequence of independently and identically distributed (i.i.d.) random variables. Its pdf has the form:

$f_{\text {GEVD }}(x)= \begin{cases}\frac{1}{\sigma}\left(1+\xi\left(\frac{x-\mu}{\sigma}\right)\right)^{\left(-\frac{1}{\xi}\right)-1} \exp \left(-\left(1+\xi\left(\frac{x-\mu}{\sigma}\right)\right)^{-\frac{1}{\xi}}\right) & \text { for } \xi \neq 0 \\ \frac{1}{\sigma} \exp \left(-\left(\frac{x-\mu}{\sigma}\right)\right) \exp \left(-\exp \left(-\left(\frac{x-\mu}{\sigma}\right)\right)\right) & \text { for } \xi=0\end{cases}$

where $\mu \in \mathbb{R}, \sigma>0$ and $\xi \in \mathbb{R}$ are the location, scale and shape parameters, respectively. When $\xi \neq 0$, the condition $1+\xi\left(\frac{x-\mu}{\sigma}\right)>0$ must hold (Coles, 2001). Parameter estimates for the vector $(\mu, \sigma, \xi)^{T}$ are obtained by a maximization of the $\log$-likelihood function $l=\ln \prod_{i=1}^{m} f\left(x_{i} ; \mu, \sigma, \xi\right)$, where $m$ denotes the number of block maxima. The maximum likelihood method offers the advantage of estimating the three parameters simultaneously.

\subsubsection{Generalized Pareto Distribution (GPD)}

The two-parameter generalized Pareto distribution (GPD) is used to model peaks-over-threshold (POT). It is characterized by a scale parameter $\sigma>0$ anda shape parameter $\xi \in \mathbb{R}$. Its pdf has the form:

$f_{G P D}(x)= \begin{cases}\frac{1}{\sigma}\left(1-\xi \frac{x}{\sigma}\right)^{\frac{1}{\xi}-1} & \text { for } \xi \neq 0 \\ \frac{1}{\sigma} \exp \left(-\frac{x}{\sigma}\right) & \text { for } \xi=0\end{cases}$

where $0 \leq x<\infty$ for $\xi \leq 0$ and $0 \leq x<\xi / k$ for $\xi>0$ (Hosking \& Wallis, 1987). For peaks-over-threshold, we consider a random variable $X$ and define the excess distribution function $F_{u}$ above a threshold $u$ as $F_{u}(x)=P(X-$ $u \leq x \mid X>u)$, where $x$ represents the magnitude of the exceedance above $u$. Estimates for the parameter vector $(\sigma, \xi)^{T}$ are obtained by maximizing the log-likelihood function $l=\ln \prod_{i=1}^{k} f\left(x_{i} ; \sigma, \xi\right)$, where $k$ denotes the number of observations satisfying $x_{i}-u \geq 0$.

\subsection{Burr XII Distribution (Burr)}

The Burr XII distribution is also known as the generalized Beta-II distribution with unit shape parameter, the Singh-Maddala distribution, as well as the Pareto-IV distribution. It is a member of a system of 12 distributions 
introduced by Burr (1942) and covers a broad range of skewness and kurtosis through different choices of parameters. This makes it suitable for modeling a wide variety of data, such as household income (Singh \& Maddala, 1976), extreme flood levels (Shao et al., 2004), and crop prices (Tejeda \& Goodwin, 2008). With $\gamma \leq x<\infty$, pdf of the four-parameter Burr distribution is given as:

$f_{\text {Burr }}(x)=\frac{\alpha k\left(\frac{x-\gamma}{\beta}\right)^{\alpha-1}}{\beta\left(1+\left(\frac{x-\gamma}{\beta}\right)^{\alpha}\right)^{k+1}}$

where $k>0, \alpha>0$ are the two shape parameters, $\beta>0$ is the scale parameter and $\gamma$ is the location parameter.

\subsection{Johnson SU Distribution (JSU)}

The Johnson SU distribution is a member of the four-parameter Johnson family of distributions that also consist of Johnson SB and the lognormal distribution (Johnson, 1949). This family covers the entire skewness-kurtosis region and Johnson SU distribution covers the area above the lognormal curve. This makes Johnson SU distribution a heavy tailed distribution and applicable to fields such as finance (Simonato, 2011) and quality control (Castagliola, 1998).

The pdf of Johnson SU distribution is given as:

$f_{J S U}(x)=\frac{\delta}{\lambda \sqrt{2 \pi} \sqrt{z^{2}+1}} \exp \left(-\frac{1}{2}\left(\gamma+\delta \sinh ^{-1}(z)\right)^{2}\right)$

where $\sinh ^{-1}(z)=\ln \left(z+\sqrt{z^{2}+1}\right), z=\frac{x-\xi}{\lambda}$ and $-\infty<x<\infty . \gamma$ and $\delta$ are the shape parameters, $\lambda$ is the scale parameter and $\xi$ is the location parameter.

\subsection{Hyperbolic Secant Distribution (HSec)}

The pdf of two-parameter hyperbolic secant distribution can be expressed as:

$f_{H S e c}(x)=\frac{1}{2 \sigma} \operatorname{sech}\left(\frac{\pi(x-\mu)}{2 \sigma}\right)$

where $-\infty<x<\infty, \sigma>0$ is the scale parameter and $\mu$ is the location parameter.

Theoretical aspects of the hyperbolic secant distribution have been considered by many authors (for example, see Baten, 1934; Harkness \& Harkness, 1968). It shares many properties with the standard normal distribution, but it is leptokurtic and has finite moments. Hence, it is suitable for the depiction of heavy-tailed data. Some examples of its application include the modeling of asset returns (Palmitesta \& Provasi, 2004) and exchange rate data (Fischer, 2006).

\subsection{Dagum Distribution (Dag)}

A series of papers by Dagum $(1975,1977)$, proposed the Dagum distribution as a new model for personal income distributions. Its heavy tails are suitable for the modeling of extreme data and have recently been applied to estimating the Tropospheric Ozone levels (Monroy et al., 2013). The pdf of four-parameter Dagum distribution is given as:

$f_{\text {Dag }}(x)=\frac{\alpha k\left(\frac{x-\gamma}{\beta}\right)^{\alpha k-1}}{\beta\left(1+\left(\frac{x-\gamma}{\beta}\right)^{\alpha}\right)^{k+1}}$

where $k>0$ and $\alpha>0$ are the two shape parameters, $\beta>0$ is the scale parameter, $\gamma$ is the location parameter and $\gamma \leq x<\infty$. It is also inversely proportional to the Burr distribution. 


\section{VALUE-AT-RISK ESTIMATION AND BACKTESTING}

The amount of market risk capital, set-aside by financial institutes as per the Basel Accord, is directly linked to the level of portfolio risk, and VaR is a common benchmark measure for evaluating such risk. VaR is intended to assess the maximum possible loss for a portfolio over a specified time period and its calculations focus on the tails of a distribution. Hence, the accuracy of VaR estimation is dependent on how well the corresponding model portrays the extreme data observations (McNeil et al., 2005; Jorion, 2006). This provides procedures for testing the robustness of a model.

For a random variable $X$ (usually the log-return of some risky financial instrument) with distribution function $F$ over a specified time period, the $\operatorname{VaR}$ (for a given probability $p$ ) can be defined as the $p$-th quantile of $F$, i.e.,

$\operatorname{VaR}_{p}=F^{-1}(1-p)$

where $F^{-1}$ is the quantile function.

A separate treatment is required for EVT, since GEVD and GPD are fitted only to the block maxima and threshold exceedances, respectively (and not on the whole data series). For a small upper tail probability $p$, GEVD approximation to VaR can be written as:

$\widehat{\operatorname{VaR}_{p}}= \begin{cases}\hat{\mu}-\frac{\hat{\sigma}}{\hat{\xi}}\left\{1-[-n \ln (1-p)]^{-\hat{\xi}}\right\}, & \hat{\xi} \neq 0 \\ \hat{\mu}-\sigma \ln [-n \ln (1-p)], & \hat{\xi}=0\end{cases}$

where $n$ is the size of the blocks and $\hat{\mu}, \hat{\sigma}$, and $\hat{\xi}$ are the maximum likelihood estimates of the GEVD parameters (Tsay, 2013), and the GPD approximation to VaR is given by:

$\widehat{\operatorname{VaR}}_{p}= \begin{cases}u+\frac{\widehat{\beta}}{\hat{\xi}}\left\{\left(\frac{n}{N_{u}} p\right)^{-\hat{\xi}}-1\right\}, & \hat{\xi} \neq 0 \\ u-\hat{\beta} \log \left(\frac{n}{N_{u}}(1-p)\right), & \hat{\xi}=0\end{cases}$

where $\hat{\beta}$ and $\hat{\xi}$ are the estimates of the GPD parameters and $N_{u}$ is the number of exceedances above the threshold $u$ in a given sample (Tsay, 2010).

In this research, we test VaR model specifications and effectiveness by utilizing the widely accepted Kupiec likelihood ratio (LR) unconditional coverage test (Kupiec, 1995) and Christoffersen conditional coverage test (Christoffersen, 1998).

The Kupiec test utilizes the fact that a good model should have its proportion of violations of VaR estimates close to the corresponding tail probability. The method consists of calculating $x^{\alpha}$ the number of times the observed returns fall below (for long positions) or above (for short positions) the VaR estimate at level $\alpha$; i.e., $r_{t}\left\langle\operatorname{VaR}^{\alpha}\right.$ or $\left.r_{t}\right\rangle$ $V a R^{\alpha}$, and compare the corresponding failure rates to $\alpha$. The null hypothesis is that the expected proportion of violations is equal to $\alpha$. Under this null hypothesis, the Kupiec statistic, given by:

$L R_{U C}=2 \ln \left(\left(\frac{x^{\alpha}}{N}\right)^{x^{\alpha}}\left(1-\frac{x^{\alpha}}{N}\right)^{N-x^{\alpha}}\right)-2 \ln \left(\alpha^{x^{\alpha}}(1-\alpha)^{N-x^{\alpha}}\right)$

is asymptotically distributed according to a chi-square distribution with one degree of freedom. The Christoffersen test extends the Kupiec test to account for serial independence of violations (i.e., clustering of extremes). The Christoffersen test statistic can be represented by: 
$L R_{C C}=L R_{U C}+2 \ln \frac{\left\lfloor\left(1-\pi_{0}\right)^{\varphi_{00}} \pi_{0}^{\varphi_{01}}\left(1-\pi_{1}\right)^{\varphi_{10}} \pi_{1} \varphi_{11}\right\rfloor}{\ln \left[(1-\pi)^{\left(\varphi_{00}+\varphi_{10}\right)} \pi^{\left(\varphi_{01}+\varphi_{11}\right)}\right\rfloor}$

where $\varphi_{i j}$ is defined as the number of returns in state $i$ while they have been in state $j$ previously (state 1 indicates the VaR estimate is violated and state 0 indicates it is not) and $\pi_{i}$ is defined as the probability of having an exception that is conditional on state $i$ the previous day. This statistic is asymptotically chi-square distributed with two degrees of freedom.

\section{EMPIRICAL RESULTS}

In this section, we fit the various distributions from Section 3 to the eight major JSE indices introduced in Section 2. Apart from JSU, all other distributions are fitted via the maximum likelihood estimation. Estimation of the JSU parameters is performed using quantile estimation, following the procedure of Wheeler (1980). The goodness-of-fit of the models is examined by utilizing the Kolmogorov-Smirnov test (Kolmogorov, 1933; Smirnov, 1948) and the Anderson-Darling test (Anderson \& Darling, 1952). The distributions are also employed to produce VaR estimates for each index and are contrasted against historical simulated VaR estimates. Backtesting on the distributional VaR estimates is then performed using Kupiec LR unconditional coverage test and Christoffersen conditional coverage test.

Table 3 presents results from Kolmogorov-Smirnov test and Anderson-Darling test. Note that comparisons on this table are not applicable to GEVD and GPD. These two distributions are not fitted onto the whole return series but only on block maxima and sizes of exceedances, respectively. Hence, no direct comparison is obtainable using the two tests discussed here. However, these results still provide insights for the performance of other models on JSE indices. Furthermore, it must be noted that Anderson-Darling test provide more emphasis on the tails of the data (Farrel \& Stewart, 2006). This is critical for VaR estimation and risk analysis for extreme losses.

For ALSI, JSU is evidently the most robust model, with the highest $p$-value in both tests, although NIG and GHSt also provide very good data depictions. On the other hand, tests for ALSI40 show that NIG produces a slightly better fit than JSU. This is most likely as a direct cause of ALSI40 having a slightly smaller skewness and kurtosis. Burr, HSec, and Dag can all be rejected as suitable models for ALSI and ALSI40, at 5\% level of significance.

None of the distributions can be rejected as suitable models for Africa40 and RESI10. With minimal difference, Hyp and VG appear as best models for Africa40. For RESI10, however, GHS $t$ is undoubtedly the preferred model. Burr, HSec, and Dag are again rejected for all of RAFI, FINDI, and CALSI. For RAFI, the Anderson-Darling test indicates NIG as the best model, although Hyp, NIG, and JSU produced similar Kolmogorov-Smirnov test results. The FINDI and CALSI return series are best described by NIG and JSU.

The SAVI returns presented the highest kurtosis and skewness, relative to all other indices (see Table 2). These properties make SAVI distinctive from other market indices. This is confirmed by the goodness-of-fit tests, which rejected all distributions for the Kolmogorov-Smirnov test and only Hyp, NIG, and GHS $t$ were not rejected for the Anderson-Darling test at $5 \%$ level of significance. 
Table 3: Kolmogorov-Smirnov Test and Anderson-Darling Test on Major JSE Indices versus Heavy-Tailed Distributions

\begin{tabular}{|c|c|c|c|c|c|c|c|c|c|c|c|c|}
\hline Index & & ibutions & Нур & NIG & VG & GHSt & Burr & JSU & HSec & Dag & GEVD & GPD \\
\hline \multirow{4}{*}{ ALSI } & \multirow{2}{*}{$\mathrm{KS}$} & Statistic & 0.0145 & 0.0117 & 0.0157 & 0.0111 & 0.0228 & 0.0067 & 0.0294 & 0.0924 & \multirow{4}{*}{ n.a. } & \multirow{4}{*}{ n.a. } \\
\hline & & $\mathrm{p}$-value & 0.6652 & 0.8837 & 0.5647 & 0.9151 & 0.1467 & 0.9999 & 0.0261 & $<0.001$ & & \\
\hline & \multirow{2}{*}{$\mathrm{AD}$} & Statistic & 0.5585 & 0.3244 & 0.7807 & 0.3616 & 3.217 & 0.2797 & 3.728 & 41.559 & & \\
\hline & & p-value & 0.6881 & 0.9188 & 0.4951 & 0.8857 & 0.0228 & 0.9526 & 0.0129 & $<0.001$ & & \\
\hline \multirow{4}{*}{ ALSI40 } & \multirow{2}{*}{$\mathrm{KS}$} & Statistic & 0.012 & 0.0095 & 0.0131 & 0.0116 & 0.0242 & 0.0118 & 0.0264 & 0.0342 & \multirow{4}{*}{ n.a. } & \multirow{4}{*}{ n.a. } \\
\hline & & p-value & 0.8635 & 0.9785 & 0.7828 & 0.8871 & 0.1063 & 0.8742 & 0.0609 & 0.0057 & & \\
\hline & \multirow{2}{*}{$\mathrm{AD}$} & Statistic & 0.4896 & 0.3041 & 0.6753 & 0.3711 & 3.0007 & 0.4232 & 3.0957 & 6.9333 & & \\
\hline & & p-value & 0.7574 & 0.9350 & 0.5798 & 0.8768 & 0.0310 & 0.8255 & 0.0274 & $<0.001$ & & \\
\hline \multirow{4}{*}{ Africa40 } & \multirow{2}{*}{$\mathrm{KS}$} & Statistic & 0.0163 & 0.0176 & 0.0157 & 0.0189 & 0.0244 & 0.0204 & 0.0229 & 0.0268 & \multirow{4}{*}{ n.a. } & \multirow{4}{*}{ n.a. } \\
\hline & & p-value & 0.9949 & 0.9868 & 0.9970 & 0.9726 & 0.8184 & 0.9472 & 0.8719 & 0.7224 & & \\
\hline & \multirow{2}{*}{$\mathrm{AD}$} & Statistic & 0.1493 & 0.1600 & 0.1537 & 0.2299 & 0.5152 & 0.1813 & 0.3035 & 0.6450 & & \\
\hline & & $\mathrm{p}$-value & 0.9986 & 0.9977 & 0.9983 & 0.9800 & 0.3553 & 0.9947 & 0.3935 & 0.3318 & & \\
\hline \multirow{4}{*}{ RESI10 } & \multirow{2}{*}{$\mathrm{KS}$} & Statistic & 0.0139 & 0.0128 & 0.0152 & 0.0101 & 0.0172 & 0.0129 & 0.0185 & 0.0196 & \multirow{4}{*}{ n.a. } & \multirow{4}{*}{ n.a. } \\
\hline & & p-value & 0.7206 & 0.8098 & 0.6079 & 0.9608 & 0.4461 & 0.8027 & 0.3565 & 0.2893 & & \\
\hline & \multirow{2}{*}{$\mathrm{AD}$} & Statistic & 0.7400 & 0.5391 & 1.0286 & 0.2691 & 1.3495 & 0.4151 & 1.3221 & 1.1394 & & \\
\hline & & p-value & 0.5263 & 0.7073 & 0.3426 & 0.9594 & 0.2046 & 0.8336 & 0.2095 & 0.2425 & & \\
\hline \multirow{4}{*}{ RAFI } & \multirow{2}{*}{$\mathrm{KS}$} & Statistic & 0.0135 & 0.0135 & 0.015 & 0.0155 & 0.0334 & 0.0133 & 0.0383 & 0.0625 & \multirow{4}{*}{ n.a. } & \multirow{4}{*}{ n.a. } \\
\hline & & $\mathrm{p}$-value & 0.7552 & 0.7503 & 0.6237 & 0.5851 & 0.0073 & 0.7718 & 0.0013 & $<0.001$ & & \\
\hline & \multirow{2}{*}{$\mathrm{AD}$} & Statistic & 0.5545 & 0.361 & 0.7735 & 0.6913 & 5.2412 & 0.5064 & 4.5721 & 21.599 & & \\
\hline & & p-value & 0.692 & 0.8863 & 0.5005 & 0.5661 & $<0.001$ & 0.7403 & $<0.001$ & $<0.001$ & & \\
\hline \multirow{4}{*}{ FINDI } & $K S$ & Statistic & 0.0129 & 0.011 & 0.0135 & 0.0149 & 0.0295 & 0.0118 & 0.0339 & 0.0275 & & \\
\hline & KS & p-value & 0.7991 & 0.9228 & 0.7488 & 0.6335 & 0.0255 & 0.8797 & 0.0063 & 0.0452 & & \\
\hline & & Statistic & 0.6241 & 0.5492 & 0.7699 & 0.9207 & 4.509 & 0.4988 & 5.1585 & 2.5991 & n.a. & n.a. \\
\hline & AD & $\mathrm{p}$-value & 0.6255 & 0.6972 & 0.5032 & 0.4017 & $<0.001$ & 0.7480 & $<0.001$ & 0.0463 & & \\
\hline & & Statistic & 0.0124 & 0.0097 & 0.0139 & 0.0123 & 0.0257 & 0.0109 & 0.0333 & 0.0917 & & \\
\hline $\mathrm{CAISI}$ & KS & p-value & 0.8351 & 0.9724 & 0.7168 & 0.8403 & 0.0723 & 0.9267 & 0.0077 & $<0.001$ & & \\
\hline CALSI & $\Delta D$ & Statistic & 0.5192 & 0.3322 & 0.7258 & 0.4605 & 3.258 & 0.3053 & 3.8946 & 41.802 & n.a. & n.a. \\
\hline & AD & p-value & 0.7273 & 0.9121 & 0.5376 & 0.7874 & 0.0212 & 0.9341 & 0.0102 & $<0.001$ & & \\
\hline & & Statistic & 0.0462 & 0.0486 & 0.0947 & 0.0452 & 0.0687 & 0.062 & 0.0814 & 0.1002 & & \\
\hline SAVI & KS & p-value & 0.0013 & $<0.001$ & $<0.001$ & 0.0018 & $<0.001$ & $<0.001$ & $<0.001$ & $<0.001$ & $n_{-}$ & $\mathrm{n}-\mathrm{a}$ \\
\hline SAVI & $\mathrm{AD}$ & Statistic & 1.982 & 1.795 & 18.188 & 1.9718 & 16.117 & 3.5382 & 8.1412 & 53.607 & il. a. & il.a. \\
\hline & AD & p-value & 0.0940 & 0.1194 & $<0.001$ & 0.0952 & $<0.001$ & 0.0147 & $<0.001$ & $<0.001$ & & \\
\hline
\end{tabular}


Tables 4.1 to 11.2 present the distributional VaR estimates, number of VaR violations, Kupiec LR test results, and Christoffersen test results for the different models on the eight JSE indices. All VaR calculations and tests are performed at the common $0.1 \%, 1 \%$, and $5 \%$ for long positions, and at $95 \%, 99 \%$, and $99.9 \%$ for short positions of trade. These results are vital in the determination of VaR forecasting adequacy of the different models on the indices. Moreover, it allows comparison across distributions that are not necessarily fitted over the same part of the data. In our case, it allows us to compare GEVD and GPD with the other distributions.

For GEVD, three different fits are performed at block sizes 5, 10, and 21 (producing weekly, fortnightly, and monthly maxima) and the corresponding models are denoted by GEVD5, GEVD10, and GEVD21, respectively. Whereas, GPD is fitted at three different threshold levels, $85 \%, 90 \%$, and $95 \%$ quantiles (locating 15\%, 10\%, and 5\% of observations as exceedances, respectively). These models are denoted by GPD85, GPD90, and GPD95. The negative tails are fitted using the relation $\min \left\{X_{1}, \ldots \ldots, X_{n}\right\}=\max \left\{-X_{1}, \ldots,-X_{n}\right\}$, i.e., multiplying the data series by negative one and perform the block method and POT method as described in Section 3.2. Thereafter, VaR estimates are calculated as described in Section 4.

It should be noted that normality is rejected almost everywhere, as one would expect due to the leptokurtic nature of these data series. Hence, normal distribution assumption often produces underestimates for VaR and, as a result, an excess number of violations. The widely recommended standard Student's $t$-distribution is a better candidate for VaR estimation, however, it is still relatively weaker (in most cases) compared to the heavy-tailed distributions discussed here.

For ALSI (Tables 4-1 and 4-2), there is a varying pattern of VaR estimates. While certain distributions underestimate the VaR at a particular significance level, they may also overestimate VaR at another. According to the Kupiec LR test, the best models at $0.1 \%$ VaR level are NIG, JSU, GPD85, and GPD90; the best model for $1 \%$ VaR is GPD85; the best model at 5\% VaR is NIG; GPD95 is the best model at 95\% VaR level, HSec is best for 99\% VaR estimation, and GHSt, GEVD10, GPD85, and GPD95 are most robust for 99.9\% VaR estimation. Although same selections are obtained for the Christoffersen test at $0.1 \%$ and $99.9 \%$ VaR levels, we can observe that the clustering of $\mathrm{VaR}$ violations start to occur at lesser extreme VaR levels (lower $p$-values for the Christoffersen test). This may be simply caused by the increase in the number of observations that exceed the VaR estimates. Similar observations can be made for ALSI40 (Tables 5-1 and 5-2).

The Africa40 results provide a very interesting case, where most models (only exceptions being Normal, $t$, Burr and Dag at singular VaR levels) are not rejected by the Kupiec LR test and the Christoffersen test (Tables 6-1 and 6-2). Africa40 distinctly differs from other indices by the facts that it has a lower volatility level and is recorded over a shorter time period. This makes the return series easier to depict by the models under consideration. However, there does not appear to exist a clear standout preferred model, with the best models varying across different VaR levels.

Tables 7-1 and 7-2 present the VaR estimates and backtesting results for RESI10. RESI10 has the second highest kurtosis in our set of indices and the lowest magnitude for skewness (see Table 2). These are also evidenced by the high magnitude of extreme VaR estimates and relatively small differences between magnitudes of short and long positions. The Kupiec LR test stipulates that, GHSt and GPD95 are the most suitable models for $0.1 \%$ VaR estimation; $1 \%$ VaR is best predicted by $t$, GPD85 and GPD90; JSU and GPD95 best describe the 5\% VaR; JSU, GPD90, and GPD95 are more robust for 95\% VaR estimation; $99 \%$ VaR is best modeled by GHSt, and $99.9 \%$ VaR can be depicted by various distributions.

Results for RAFI are presented in Tables 8-1 and 8-2. It is interesting to note that Dag is a particularly bad model for RAFI, with the distribution rejected at all VaR levels for the Kupiec LR test and is also rejected 5 out of 6 VaR levels for the Christoffersen test, at 5\% level of significance. We may also observe that GPD90 and GPD95 produce very good VaR estimates for RAFI. Although they do not always give the highest $p$-values for the Kupiec LR test, they are never rejected by the Kupiec LR test, at all levels of significance.

GPD95 standout as a good VaR model for FINDI (Tables 9-1 and 9-2), because it has relatively high $p$-values for all VaR levels in the Kupiec LR test. However, the model with the highest Kupiec test $p$-value still varies across VaR levels. For example, the standard Student's $t$-distribution is the clear best model (by far) for $99 \%$ VaR estimation. 
Such irregularity is also presented in the Christoffersen test, where it is also evidenced that extreme clustering is common for $95 \%$ and $99 \%$ VaR estimates. Results for CALSI (Tables 10-1 and 10-2) depict similar observations.

Tables 11-1 and 11-2 illustrate the VaR results for SAVI returns. SAVI portrays an extreme case under our analyses. It has the highest kurtosis value and the highest magnitude in skewness. Given its construct as the "fear" gauge to measure current market sentiment, such characteristics can be expected. Hence, the extreme VaR estimates are high in magnitude (relative to other indices) and there is a relatively large difference between the estimates of short and long positions. These properties make SAVI very suitable for our model fitting, as most of our distributions are heavy-tailed and cater for substantial skewness. Hence, none of Hyp, NIG, VG, GHSt, JSU, GEVD5, GPD85, GPD90, and GPD95 can be rejected by the Kupiec LR test as good models for SAVI. Interestingly, this is also the only index that produces the exact same best model selections from both the Kupiec LR test and the Christoffersen test. In particular, NIG, GHSt, JSU, and GEVD5 give the best estimation for $0.1 \%$ VaR; GHSt, JSU, GEVD10, GEVD21, GPD90, and GPD95 are all best suited for 1\% VaR estimation; 5\% VaR is best described by GPD95; both GHS $t$ and GPD95 are most robust for the 95\% VaR estimation; GHS $t$ is most suitable for 99\% VaR estimation; and NIG, all GEVDs, and all GPDs are appropriate for the $99.9 \%$ VaR estimation. Hence, there is again no one best model for the return series. 
Table 4-1: VaR Estimates for ALSI Using Heavy-Tailed Distributions

\begin{tabular}{|c|c|c|c|c|c|c|}
\hline \multirow[b]{2}{*}{ Distr } & \multicolumn{6}{|c|}{ VaR Estimates } \\
\hline & $0.1 \%$ & $1 \%$ & $5 \%$ & $95 \%$ & $99 \%$ & $99.9 \%$ \\
\hline Historical & -0.06345016 & -0.03448587 & -0.02034420 & 0.01874104 & 0.03419627 & 0.06050109 \\
\hline Normal & -0.03899048 & -0.02920541 & -0.02047572 & 0.0216642 & 0.03039389 & 0.04017896 \\
\hline$t$ & -0.05202774 & -0.03463711 & -0.02256283 & 0.02375131 & 0.03582559 & 0.05321622 \\
\hline Hyp & -0.05562657 & -0.03507477 & -0.02064146 & 0.02012801 & 0.03228777 & 0.04958697 \\
\hline NIG & -0.06326095 & -0.03681981 & -0.02041068 & 0.01989789 & 0.03304091 & 0.05378207 \\
\hline VG & -0.05447578 & -0.03481429 & -0.02073931 & 0.0202484 & 0.03227197 & 0.04907236 \\
\hline $\mathrm{GHS} t$ & -0.08117427 & -0.03733324 & -0.01966119 & 0.01965385 & 0.03293994 & 0.05886682 \\
\hline Burr & -0.04659 & -0.03061 & -0.01921 & 0.02001 & 0.03036 & 0.04473 \\
\hline JSU & -0.06429056 & -0.03613174 & -0.02026164 & 0.01883265 & 0.03023169 & 0.04984987 \\
\hline $\mathrm{HSec}$ & -0.05207 & -0.03328 & -0.02014 & 0.02133 & 0.03447 & 0.05325 \\
\hline Dag & -0.0427 & -0.03022 & -0.02055 & 0.02856 & 0.04494 & 0.068 \\
\hline GEVD5 & -0.052992383 & -0.033190558 & -0.019443917 & 0.01949556 & 0.032182857 & 0.051476981 \\
\hline GEVD10 & -0.051970393 & -0.032680905 & -0.018351698 & 0.017052601 & 0.030870653 & 0.062556659 \\
\hline GEVD21 & -0.056106105 & -0.030348202 & -0.015184599 & 0.015056389 & 0.028041325 & 0.073459841 \\
\hline GPD85 & -0.0646011 & -0.034111455 & -0.019248426 & 0.019230044 & 0.034125031 & 0.064791175 \\
\hline GPD90 & -0.05995494 & -0.036213694 & -0.020467578 & 0.019166717 & 0.034150989 & 0.065676166 \\
\hline GPD95 & -0.059731903 & -0.036444093 & -0.020340216 & 0.018737091 & 0.035138964 & 0.060475574 \\
\hline
\end{tabular}

\begin{tabular}{|c|c|c|c|c|c|c|c|c|c|c|c|c|c|c|c|c|c|c|}
\hline \multirow[b]{2}{*}{ Distr } & \multicolumn{6}{|c|}{ Number of Violations } & \multicolumn{6}{|c|}{$p$-value of Kupiec Test } & \multicolumn{6}{|c|}{$p$-value of Christoffersen Test } \\
\hline & $0.1 \%$ & $1 \%$ & $5 \%$ & $95 \%$ & $99 \%$ & $99.9 \%$ & $0.1 \%$ & $1 \%$ & $5 \%$ & $95 \%$ & $99 \%$ & $99.9 \%$ & $0.1 \%$ & $1 \%$ & $5 \%$ & $95 \%$ & $99 \%$ & $99.9 \%$ \\
\hline Normal & 17 & 58 & 123 & 91 & 39 & 17 & $<0.001$ & $<0.001$ & 0.8504 & 0.0011 & 0.0094 & $<0.001$ & $<0.001$ & $<0.001$ & 0.0045 & $<0.001$ & 0.0027 & $<0.001$ \\
\hline$t$ & 5 & 24 & 102 & 76 & 21 & 4 & 0.1645 & 0.8381 & 0.0291 & $<0.001$ & 0.4072 & 0.3834 & 0.3768 & 0.7761 & 0.0012 & $<0.001$ & .0290 & $\overline{796}$ \\
\hline Hyp & 5 & 24 & 123 & 111 & 33 & 9 & 0.1645 & 0.8381 & 0.8504 & 0.1892 & 0.1258 & 0.0015 & 0.3768 & 0.7761 & 0.0045 & 0.0024 & 0.0651 & $<0$. \\
\hline NIG & 3 & 21 & 125 & 115 & 29 & 4 & 0.7596 & 0.4072 & 0.9963 & 0.3502 & 0.4342 & 0.3834 & 0.9508 & 0.5937 & 0.0063 & $<0.001$ & 0.0996 & 0.6796 \\
\hline VG & 5 & 24 & 123 & 109 & 33 & 11 & 0.1645 & 0.8381 & 0.8504 & 0.1325 & 0.1258 & $<0.001$ & 0.3768 & 0.7761 & 0.0045 & 0.0040 & 0.0651 & $<0.001$ \\
\hline GHSt & 0 & 20 & 134 & 117 & 29 & 3 & 0.0253 & 0.2968 & 0.4167 & 0.4555 & 0.4342 & 0.7596 & 0.0819 & 0.4939 & 0.0026 & 0.0012 & 0.0996 & 0.9508 \\
\hline Burr & 8 & 45 & 140 & 114 & 39 & 14 & 0.0058 & $<0.001$ & 0.1780 & 0.3037 & 0.0094 & $<0.001$ & 0.0216 & $<0.001$ & $<0.001$ & $<0.001$ & 0.0027 & $<0.001$ \\
\hline JSU & 3 & 21 & 127 & 124 & 39 & 8 & 0.7596 & 0.4072 & 0.8584 & 0.9232 & 0.0094 & 0.0058 & 0.9508 & 0.5937 & 0.0086 & $<0.001$ & 0.0027 & 0.0013 \\
\hline $\mathrm{HSec}$ & 5 & 30 & 131 & 95 & 25 & 4 & 0.1645 & 0.3309 & 0.5879 & 0.0041 & 0.9984 & 0.3834 & 0.3768 & 0.0949 & 0.0052 & $<0.001$ & 0.0792 & 0.6796 \\
\hline Dag & 11 & 48 & 123 & 47 & 14 & 1 & $<0.001$ & $<0.001$ & 0.8504 & $<0.001$ & 0.0158 & 0.2795 & $<0.001$ & $<0.001$ & 0.0045 & $<0.001$ & $<0.001$ & 0.5570 \\
\hline GEVD5 & 5 & 30 & 137 & 118 & 33 & 7 & 0.1645 & 0.3309 & 0.2799 & 0.5139 & 0.1258 & 0.0199 & 0.3768 & 0.0949 & 0.0012 & 0.0015 & 0.0651 & 0.0030 \\
\hline GEVD10 & 5 & 31 & 151 & 163 & 36 & 3 & 0.1645 & 0.2459 & 0.0209 & $<0.001$ & 0.0382 & 0.7596 & 0.3768 & 0.0116 & $<0.001$ & $<0.001$ & 0.0324 & 0.9508 \\
\hline GEVD21 & 5 & 47 & 219 & 208 & 50 & 0 & 0.1645 & $<0.001$ & $<0.001$ & $<0.001$ & $<0.001$ & 0.0253 & 0.3768 & $<0.001$ & $<0.001$ & $<0.001$ & $<0.001$ & 0.0819 \\
\hline GPD85 & 3 & 26 & 139 & 121 & 26 & 3 & 0.7596 & 0.8433 & 0.2083 & 0.7088 & 0.8433 & 0.7596 & 0.9508 & 0.0897 & $<0.001$ & $<0.001$ & 0.0897 & 0.9508 \\
\hline GPD90 & 3 & 21 & 123 & 121 & 26 & 1 & 0.7596 & 0.4072 & 0.8504 & 0.7088 & 0.8433 & 0.2795 & 0.9508 & 0.5937 & 0.0045 & $<0.001$ & 0.0897 & 0.5570 \\
\hline GPD95 & 4 & 21 & 126 & 126 & 23 & 3 & 0.3834 & 0.4072 & 0.9306 & 0.9306 & 0.6822 & 0.7596 & 0.6796 & 0.5937 & 0.0074 & $<0.001$ & 0.0533 & 0.9508 \\
\hline
\end{tabular}


Table 5-1: VaR Estimates for ALSI40 using Heavy-Tailed Distributions

\begin{tabular}{|c|c|c|c|c|c|c|}
\hline \multirow[b]{2}{*}{ Distr } & \multicolumn{6}{|c|}{ VaR Estimates } \\
\hline & $0.1 \%$ & $1 \%$ & $5 \%$ & $95 \%$ & $99 \%$ & $99.9 \%$ \\
\hline Historical & -0.0672932 & -0.03807286 & -0.02252051 & 0.02065497 & 0.03815264 & 0.0655473 \\
\hline Normal & -0.04273466 & -0.03202627 & -0.02247285 & 0.02364338 & 0.03319679 & 0.04390518 \\
\hline$t$ & -0.05674717 & -0.03787282 & -0.02472151 & 0.02589203 & 0.03904335 & 0.05791769 \\
\hline Hyp & -0.06070554 & -0.03832927 & -0.02263214 & 0.02212525 & 0.03565813 & 0.05487895 \\
\hline NIG & -0.06838663 & -0.04002478 & -0.02231742 & 0.02190274 & 0.03646936 & 0.05947935 \\
\hline VG & -0.05960987 & -0.03810711 & -0.02271426 & 0.02225466 & 0.03564405 & 0.05434605 \\
\hline $\mathrm{GHS} t$ & -0.08571362 & -0.04032656 & -0.02152649 & 0.02162928 & 0.03640946 & 0.06547592 \\
\hline Burr & -0.05066 & -0.03335 & -0.021 & 0.02197 & 0.03354 & 0.04965 \\
\hline JSU & -0.07312378 & -0.04060119 & -0.02254403 & 0.02062865 & 0.03333209 & 0.05552125 \\
\hline $\mathrm{HSec}$ & -0.05704 & -0.03649 & -0.0221 & 0.02327 & 0.03766 & 0.05821 \\
\hline Dag & -0.05187 & -0.03432 & -0.02177 & 0.02363 & 0.03646 & 0.05443 \\
\hline GEVD5 & -0.057617192 & -0.036008593 & -0.021176983 & 0.021390916 & 0.035509621 & 0.057215649 \\
\hline GEVD10 & -0.056102025 & -0.035485719 & -0.020128267 & 0.018765037 & 0.034137702 & 0.069169123 \\
\hline GEVD21 & -0.059423767 & -0.033196529 & -0.016748401 & 0.016705164 & 0.031174525 & 0.076407297 \\
\hline GPD85 & -0.064995441 & -0.039019548 & -0.022317858 & 0.021190722 & 0.037584242 & 0.07100962 \\
\hline GPD90 & -0.065068043 & -0.038968102 & -0.022337789 & 0.021191447 & 0.037707634 & 0.070352143 \\
\hline GPD95 & -0.064217893 & -0.039220344 & -0.022516436 & 0.020650686 & 0.038574441 & 0.066755763 \\
\hline
\end{tabular}

Table 5-2: VaR Backtesting for ALSI40 versus Heavy-Tailed Distributions

\begin{tabular}{|c|c|c|c|c|c|c|c|c|c|c|c|c|c|c|c|c|c|c|}
\hline & \multicolumn{6}{|c|}{ Number of Violations } & \multicolumn{6}{|c|}{$p$-value of Kupiec Test } & \multicolumn{6}{|c|}{$p$-value of Christoffersen Test } \\
\hline Distr & $0.1 \%$ & $1 \%$ & $5 \%$ & $95 \%$ & $99 \%$ & $99.9 \%$ & $0.1 \%$ & $1 \%$ & $5 \%$ & $95 \%$ & $99 \%$ & $99.9 \%$ & $0.1 \%$ & $1 \%$ & $5 \%$ & $95 \%$ & $99 \%$ & $99.9 \%$ \\
\hline Normal & 16 & 51 & 126 & 92 & 40 & 16 & $<0.001$ & $<0.001$ & 0.9306 & 0.0015 & 0.0056 & $<0.001$ & $<0.001$ & $<0.001$ & 0.0196 & $<0.001$ & 0.0082 & $<0.001$ \\
\hline$t$ & 6 & 26 & 104 & 76 & 23 & 4 & 0.0611 & 0.8433 & 0.0470 & $<0.001$ & 0.6822 & 0.3834 & 0.1706 & 0.7462 & 0.0024 & $<0.001$ & 0.0533 & 0.6796 \\
\hline Hyp & 5 & 24 & 125 & 114 & 31 & 9 & 0.1645 & 0.8381 & 0.9963 & 0.3037 & 0.2459 & 0.0015 & 0.3768 & 0.7761 & 0.0171 & 0.0018 & 0.0869 & $<0.001$ \\
\hline NIG & 3 & 20 & 126 & 115 & 30 & 4 & 0.7596 & 0.2968 & 0.9306 & 0.3502 & 0.3309 & 0.3834 & 0.9508 & 0.4939 & 0.0196 & 0.0023 & 0.0949 & 0.6796 \\
\hline VG & 5 & 25 & 125 & 110 & 31 & 11 & 0.1645 & 0.9984 & 0.9963 & 0.1590 & 0.2459 & $<0.001$ & 0.3768 & 0.7768 & 0.0171 & 0.0018 & 0.0869 & $<0.001$ \\
\hline GHSt & 0 & 20 & 135 & 116 & 30 & 3 & 0.0253 & 0.2968 & 0.3672 & 0.4008 & 0.3309 & 0.7596 & 0.0819 & 0.4939 & 0.0075 & 0.0030 & 0.0949 & 0.9508 \\
\hline Burr & 9 & 43 & 140 & 115 & 39 & 14 & 0.0015 & 0.0010 & 0.1780 & 0.3502 & 0.0094 & $<0.001$ & 0.0063 & $<0.001$ & 0.0038 & 0.0023 & 0.0121 & $<0.001$ \\
\hline JSU & 2 & 20 & 125 & 126 & 39 & 8 & 0.7425 & 0.2968 & 0.9963 & 0.9306 & 0.0094 & 0.0058 & 0.9460 & 0.4939 & 0.0171 & 0.0026 & 0.0121 & 0.0013 \\
\hline HSec & 5 & 28 & 128 & 94 & 26 & 4 & 0.1645 & 0.5555 & 0.7874 & 0.0029 & 0.8433 & 0.3834 & 0.3768 & 0.6120 & 0.0248 & $<0.001$ & 0.0897 & 0.6796 \\
\hline Dag & 7 & 35 & 131 & 93 & 30 & 11 & 0.0199 & 0.0583 & 0.5879 & 0.0021 & 0.3309 & $<0.001$ & 0.0653 & 0.0074 & 0.0136 & $<0.001$ & 0.0949 & $<0.001$ \\
\hline GEVD5 & 5 & 29 & 138 & 120 & 31 & 5 & 0.1645 & 0.4342 & 0.2422 & 0.6410 & 0.2459 & 0.1645 & 0.3768 & 0.5241 & 0.0035 & $<0.001$ & 0.0869 & 0.3768 \\
\hline GEVD10 & 6 & 31 & 153 & 169 & 36 & 2 & 0.0611 & 0.2459 & 0.0131 & $<0.001$ & 0.0382 & 0.7425 & 0.1706 & 0.3583 & $<0.001$ & $<0.001$ & 0.0324 & 0.9460 \\
\hline GEVD21 & 5 & 44 & 216 & 211 & 49 & 1 & 0.1645 & $<0.001$ & $<0.001$ & $<0.001$ & $<0.001$ & 0.2795 & 0.3768 & $<0.001$ & $<0.001$ & $<0.001$ & $<0.001$ & 0.5570 \\
\hline GPD85 & 3 & 20 & 126 & 122 & 26 & 2 & 0.7596 & 0.2968 & 0.9306 & 0.7788 & 0.8433 & 0.7425 & 0.9508 & 0.4939 & 0.0196 & 0.0012 & 0.0897 & 0.9460 \\
\hline GPD90 & 3 & 21 & 126 & 122 & 26 & 2 & 0.7596 & 0.4072 & 0.9306 & 0.7788 & 0.8433 & 0.7425 & 0.9508 & 0.5937 & 0.0196 & 0.0012 & 0.0897 & 0.9460 \\
\hline GPD95 & 3 & 20 & 126 & 126 & 24 & 3 & 0.7596 & 0.2968 & 0.9306 & 0.9306 & 0.8381 & 0.7596 & 0.9508 & 0.4939 & 0.0196 & 0.0026 & 0.0666 & 0.9508 \\
\hline
\end{tabular}


Table 6-1: VaR Estimates for Africa40 using Heavy-Tailed Distributions

\begin{tabular}{|c|c|c|c|c|c|c|}
\hline \multirow[b]{2}{*}{ Distr } & \multicolumn{6}{|c|}{ VaR Estimates } \\
\hline & $0.1 \%$ & $1 \%$ & $5 \%$ & $95 \%$ & $99 \%$ & $99.9 \%$ \\
\hline Historical & -0.03547472 & -0.02000097 & -0.01118234 & 0.01314262 & 0.02136312 & 0.02708866 \\
\hline Normal & -0.02324872 & -0.01733864 & -0.012066 & 0.01338608 & 0.01865872 & 0.02456881 \\
\hline$t$ & -0.02955974 & -0.02001702 & -0.0131098 & 0.01442988 & 0.02133711 & 0.03087983 \\
\hline Hyp & -0.03094495 & -0.01967227 & -0.01174142 & 0.01333317 & 0.02164537 & 0.03350001 \\
\hline NIG & -0.03253211 & -0.01974817 & -0.01154204 & 0.01320432 & 0.02205369 & 0.03580763 \\
\hline VG & -0.03025697 & -0.01950127 & -0.01176971 & 0.01337838 & 0.02146328 & 0.03271498 \\
\hline $\mathrm{GHS} t$ & -0.03579924 & -0.01971428 & -0.0113911 & 0.01291395 & 0.0218945 & 0.04039046 \\
\hline Burr & -0.02678 & -0.01766 & -0.01114 & 0.01314 & 0.02038 & 0.03061 \\
\hline JSU & -0.03412504 & -0.01944475 & -0.0112097 & 0.01311631 & 0.02240027 & 0.0391158 \\
\hline $\mathrm{HSec}$ & -0.03116 & -0.01981 & -0.01187 & 0.01319 & 0.02113 & 0.03248 \\
\hline Dag & -0.02799 & -0.01839 & -0.01154 & 0.01272 & 0.01949 & 0.02896 \\
\hline GEVD5 & -0.032793816 & -0.019925227 & -0.011480848 & 0.013736753 & 0.020792893 & 0.029031259 \\
\hline GEVD10 & -0.03618093 & -0.019487097 & -0.010664574 & 0.013553361 & 0.020553032 & 0.028893693 \\
\hline GEVD21 & -0.037918587 & -0.019508359 & -0.010685402 & 0.013744118 & 0.020979166 & 0.0282553 \\
\hline GPD85 & -0.036001712 & -0.020271111 & -0.011352657 & 0.01373642 & 0.020891273 & 0.028271379 \\
\hline GPD90 & -0.035386952 & -0.020449192 & -0.011397043 & 0.01362005 & 0.020854378 & 0.02858942 \\
\hline GPD95 & -0.038557689 & -0.019778566 & -0.011189117 & 0.01315441 & 0.021940674 & 0.027093932 \\
\hline
\end{tabular}

Table 6-2: VaR Backtesting for Africa40 versus Heavy-Tailed Distributions

\begin{tabular}{|c|c|c|c|c|c|c|c|c|c|c|c|c|c|c|c|c|c|c|}
\hline \multirow[b]{2}{*}{ Distr } & \multicolumn{6}{|c|}{ Number of Violations } & \multicolumn{6}{|c|}{$p$-value of Kupiec Test } & \multicolumn{6}{|c|}{$p$-value of Christoffersen Test } \\
\hline & $0.1 \%$ & $1 \%$ & $5 \%$ & $95 \%$ & $99 \%$ & $99.9 \%$ & $0.1 \%$ & $1 \%$ & $5 \%$ & $95 \%$ & $99 \%$ & $99.9 \%$ & $0.1 \%$ & $1 \%$ & $\mathbf{5 \%}$ & $95 \%$ & $99 \%$ & $99.9 \%$ \\
\hline Normal & 5 & 8 & 27 & 32 & 12 & 3 & $<0.001$ & 0.5933 & 0.2729 & 0.8646 & 0.0575 & 0.0355 & 0.0029 & 0.7858 & 0.0413 & 0.9238 & 0.0745 & 0.1082 \\
\hline$t$ & 2 & 7 & 21 & 30 & 8 & 0 & 0.1844 & 0.8737 & 0.0225 & 0.5926 & 0.5933 & 0.2508 & 0.4120 & 0.9159 & $<0.001$ & 0.7519 & 0.7858 & 0.5172 \\
\hline Hyp & 2 & 8 & 29 & 32 & 5 & 0 & 0.1844 & 0.5933 & 0.4715 & 0.8646 & 0.5156 & 0.2508 & 0.4120 & 0.7858 & 0.0922 & 0.9238 & 0.7791 & 0.5172 \\
\hline NIG & 2 & 8 & 30 & 32 & 5 & 0 & 0.1844 & 0.5933 & 0.5926 & 0.8646 & 0.5156 & 0.2508 & 0.4120 & 0.7858 & 0.1276 & 0.9238 & 0.7791 & 0.5172 \\
\hline VG & 2 & 8 & 29 & 32 & 5 & 0 & 0.1844 & 0.5933 & 0.4715 & 0.8646 & 0.5156 & 0.2508 & 0.4120 & 0.7858 & 0.0922 & 0.9238 & 0.7791 & 0.5172 \\
\hline $\mathrm{GHS} t$ & 1 & 8 & 31 & 34 & 5 & 0 & 0.6964 & 0.5933 & 0.7249 & 0.8519 & 0.5156 & 0.2508 & 0.9253 & 0.7858 & 0.1681 & 0.9652 & 0.7791 & 0.5172 \\
\hline Burr & 3 & 8 & 34 & 33 & 9 & 0 & 0.0355 & 0.5933 & 0.8519 & 0.9929 & 0.3714 & 0.2508 & 0.1082 & 0.7858 & 0.2925 & 0.9631 & 0.5920 & 0.5172 \\
\hline JSU & 1 & 8 & 33 & 34 & 4 & 0 & 0.6964 & 0.5933 & 0.9929 & 0.8519 & 0.2741 & 0.2508 & 0.9253 & 0.7858 & 0.2542 & 0.9652 & 0.5366 & 0.5172 \\
\hline $\mathrm{HSec}$ & 2 & 7 & 28 & 32 & 8 & 0 & 0.1844 & 0.8737 & 0.3643 & 0.8646 & 0.5933 & 0.2508 & 0.4120 & 0.9159 & 0.0634 & 0.9238 & 0.7858 & 0.5172 \\
\hline Dag & 3 & 8 & 30 & 35 & 10 & 0 & 0.0355 & 0.5933 & 0.5926 & 0.7167 & 0.2148 & 0.2508 & 0.1082 & 0.7858 & 0.1276 & 0.9310 & 0.1524 & 0.5172 \\
\hline GEVD5 & 1 & 7 & 30 & 31 & 9 & 0 & 0.6964 & 0.8737 & 0.5926 & 0.7249 & 0.3714 & 0.2508 & 0.9253 & 0.9159 & 0.1276 & 0.8510 & 0.5920 & 0.5172 \\
\hline GEVD10 & 1 & 8 & 38 & 31 & 9 & 0 & 0.6964 & 0.5933 & 0.3777 & 0.7249 & 0.3714 & 0.2508 & 0.9253 & 0.7858 & 0.3395 & 0.8510 & 0.5920 & 0.5172 \\
\hline GEVD21 & 1 & 8 & 38 & 31 & 8 & 0 & 0.6964 & 0.5933 & 0.3777 & 0.7249 & 0.5933 & 0.2508 & 0.9253 & 0.7858 & 0.3395 & 0.8510 & 0.7858 & 0.5172 \\
\hline GPD85 & 1 & 7 & 32 & 31 & 9 & 0 & 0.6964 & 0.8737 & 0.8646 & 0.7249 & 0.3714 & 0.2508 & 0.9253 & 0.9159 & 0.2115 & 0.8510 & 0.5920 & 0.5172 \\
\hline GPD90 & 1 & 6 & 31 & 31 & 9 & 0 & 0.6964 & 0.8146 & 0.7249 & 0.7249 & 0.3714 & 0.2508 & 0.9253 & 0.9206 & 0.1681 & 0.8510 & 0.5920 & 0.5172 \\
\hline GPD95 & 1 & 8 & 33 & 33 & 5 & 1 & 0.6964 & 0.5933 & 0.9929 & 0.9929 & 0.5156 & 0.6964 & 0.9253 & 0.7858 & 0.2542 & 0.9631 & 0.7791 & 0.9253 \\
\hline
\end{tabular}


Table 7-1: VaR Estimates for RESI10 using Heavy-Tailed Distributions

\begin{tabular}{|c|c|c|c|c|c|c|}
\hline \multirow[b]{2}{*}{ Distr } & \multicolumn{6}{|c|}{ VaR Estimates } \\
\hline & $0.1 \%$ & $1 \%$ & $5 \%$ & $95 \%$ & $99 \%$ & $99.9 \%$ \\
\hline Historical & -0.09396051 & -0.05199949 & -0.02953367 & 0.02855521 & 0.05007265 & 0.1035711 \\
\hline Normal & -0.05854103 & -0.0439762 & -0.03098228 & 0.03174192 & 0.04473584 & 0.05930067 \\
\hline$t$ & -0.07705341 & -0.05171818 & -0.03396445 & 0.03472409 & 0.05247782 & 0.07781305 \\
\hline Hyp & -0.07685876 & -0.04944006 & -0.03011218 & 0.03037305 & 0.04905526 & 0.07545615 \\
\hline NIG & -0.08396262 & -0.05101173 & -0.02987517 & 0.03013175 & 0.050342 & 0.08170223 \\
\hline VG & -0.07532223 & -0.04920109 & -0.03024126 & 0.03059851 & 0.04908793 & 0.07458935 \\
\hline $\mathrm{GHS} t$ & -0.09618747 & -0.0508996 & -0.02908365 & 0.02948061 & 0.05009455 & 0.09082008 \\
\hline Burr & -0.06782 & -0.04501 & -0.02872 & 0.02999 & 0.04671 & 0.07016 \\
\hline JSU & -0.08203889 & -0.04916091 & -0.02942634 & 0.02866594 & 0.04644286 & 0.0757165 \\
\hline $\mathrm{HSec}$ & -0.078 & -0.05005 & -0.03048 & 0.03124 & 0.05081 & 0.07876 \\
\hline Dag & -0.07052 & -0.04707 & -0.02977 & 0.02909 & 0.0453 & 0.06878 \\
\hline GEVD5 & -0.076419809 & -0.048126721 & -0.028759959 & 0.029437468 & 0.048841893 & 0.077910687 \\
\hline GEVD10 & -0.074494929 & -0.047512228 & -0.027557696 & 0.026448973 & 0.046846716 & 0.086283603 \\
\hline GEVD21 & -0.08121959 & -0.043261028 & -0.022818916 & 0.024572043 & 0.044120567 & 0.087118577 \\
\hline GPD85 & -0.091362171 & -0.051871602 & -0.029227576 & 0.029141335 & 0.051280598 & 0.093332054 \\
\hline GPD90 & -0.09161431 & -0.051693318 & -0.029275834 & 0.028799235 & 0.050852149 & 0.097970781 \\
\hline GPD95 & -0.092610603 & -0.051410683 & -0.029528819 & 0.02864884 & 0.051339117 & 0.095705283 \\
\hline
\end{tabular}

Table 7-2: VaR Cacktesting for RESI10 versus Heavy-Tailed Distributions

\begin{tabular}{|c|c|c|c|c|c|c|c|c|c|c|c|c|c|c|c|c|c|c|}
\hline \multirow[b]{2}{*}{ Distr } & \multicolumn{6}{|c|}{ Number of Violations } & \multicolumn{6}{|c|}{$p$-value of Kupiec Test } & \multicolumn{6}{|c|}{$p$-value of Christoffersen Test } \\
\hline & $0.1 \%$ & $1 \%$ & $5 \%$ & $95 \%$ & $99 \%$ & $\mathbf{9 9 . 9 \%}$ & $0.1 \%$ & $1 \%$ & $5 \%$ & $95 \%$ & $99 \%$ & $99.9 \%$ & $0.1 \%$ & $1 \%$ & $5 \%$ & $95 \%$ & $99 \%$ & $99.9 \%$ \\
\hline Normal & 16 & $\overline{41}$ & 110 & 102 & 36 & 14 & $<0.001$ & 0.0033 & 0.1590 & 0.0291 & 0.0382 & $<0.001$ & $<0.001$ & 0.0013 & $<0.001$ & 0.0034 & $<0.001$ & $<0.001$ \\
\hline$t$ & 6 & 26 & 89 & 77 & 23 & 7 & 0.0611 & 0.8433 & $<0.001$ & $<0.001$ & 0.6822 & 0.0199 & 0.1706 & 0.7462 & $<0.001$ & $<0.001$ & 0.0533 & 0.0030 \\
\hline Нур & 6 & 30 & 121 & 112 & 28 & 8 & 0.0611 & 0.3309 & 0.7088 & 0.2232 & 0.5555 & 0.0058 & 0.1706 & 0.4330 & $<0.001$ & 0.0552 & 0.1004 & $<0.001$ \\
\hline NIG & 6 & 27 & 122 & 113 & 24 & 5 & 0.0611 & 0.6930 & 0.7788 & 0.2614 & 0.8381 & 0.1645 & 0.1706 & 0.6888 & 0.0012 & 0.0681 & 0.0666 & 0.0083 \\
\hline VG & 6 & 30 & 120 & 112 & 28 & 8 & 0.0611 & 0.3309 & 0.6410 & 0.2232 & 0.5555 & 0.0058 & 0.1706 & 0.4330 & $<0.001$ & 0.0552 & 0.1004 & $<0.001$ \\
\hline GHSt & 3 & 27 & 129 & 119 & 25 & 5 & 0.7596 & 0.6930 & 0.7184 & 0.5759 & 0.9984 & 0.1645 & 0.9508 & 0.6888 & 0.0041 & 0.0923 & 0.0792 & 0.0083 \\
\hline Burr & 9 & 39 & 131 & 115 & 32 & 9 & 0.0015 & 0.0094 & 0.5879 & 0.3502 & 0.1781 & 0.0015 & 0.0063 & 0.0306 & 0.0052 & 0.0999 & 0.0110 & $<0.001$ \\
\hline JSU & 6 & 30 & 126 & 124 & 32 & 7 & 0.0611 & 0.3309 & 0.9306 & 0.9232 & 0.1781 & 0.0199 & 0.1706 & 0.4330 & 0.0026 & 0.1724 & 0.0110 & 0.0030 \\
\hline $\mathrm{HSec}$ & 6 & 27 & 114 & 106 & 24 & 7 & 0.0611 & 0.6930 & 0.3037 & 0.0730 & 0.8381 & 0.0199 & 0.1706 & 0.6888 & $<0.001$ & 0.0122 & 0.0666 & 0.0030 \\
\hline Dag & 8 & 34 & 122 & 122 & 33 & 10 & 0.0058 & 0.0867 & 0.7788 & 0.7788 & 0.1258 & $<0.001$ & 0.0216 & 0.1809 & 0.0012 & 0.1389 & 0.0100 & $<0.001$ \\
\hline GEVD5 & 6 & 31 & 130 & 119 & 28 & 7 & 0.0611 & 0.2459 & 0.6517 & 0.5759 & 0.5555 & 0.0199 & 0.1706 & 0.3456 & 0.0046 & 0.0923 & 0.1004 & 0.0030 \\
\hline GEVD10 & 6 & 33 & 140 & 149 & 32 & 5 & 0.0611 & 0.1258 & 0.1780 & 0.0327 & 0.1781 & 0.1645 & 0.1706 & 0.1993 & 0.0038 & 0.0032 & 0.0110 & 0.0083 \\
\hline GEVD21 & 6 & 43 & 214 & 181 & 37 & 5 & 0.0611 & 0.0010 & $<0.001$ & $<0.001$ & 0.0245 & 0.1645 & 0.1706 & $<0.001$ & $<0.001$ & $<0.001$ & $<0.001$ & 0.0083 \\
\hline GPD85 & 4 & 26 & 128 & 122 & 24 & 5 & 0.3834 & 0.8433 & 0.7874 & 0.7788 & 0.8381 & 0.1645 & 0.6796 & 0.7462 & 0.0035 & 0.1389 & 0.0666 & 0.0083 \\
\hline GPD90 & 4 & 26 & 128 & 124 & 24 & 5 & 0.3834 & 0.8433 & 0.7874 & 0.9232 & 0.8381 & 0.1645 & 0.6796 & 0.7462 & 0.0035 & 0.1724 & 0.0666 & 0.0083 \\
\hline GPD95 & 3 & 27 & 126 & 124 & 24 & 5 & 0.7596 & 0.6930 & 0.9306 & 0.9232 & 0.8381 & 0.1645 & 0.9508 & 0.6888 & 0.0026 & 0.1724 & 0.0666 & 0.0083 \\
\hline
\end{tabular}


Table 8-1: VaR Estimates for RAFI using Heavy-Tailed Distributions

\begin{tabular}{|c|c|c|c|c|c|c|}
\hline \multirow[b]{2}{*}{ Distr } & \multicolumn{6}{|c|}{$\begin{array}{ll}\text { VaR Estimates } \\
\end{array}$} \\
\hline & $0.1 \%$ & $1 \%$ & $5 \%$ & $95 \%$ & $99 \%$ & $99.9 \%$ \\
\hline Historical & -0.05357548 & -0.03292602 & -0.02004062 & 0.01853224 & 0.032919 & 0.04985304 \\
\hline Normal & -0.03637012 & -0.02723749 & -0.01908988 & 0.02024026 & 0.02838787 & 0.0375205 \\
\hline$t$ & -0.04815221 & -0.03215902 & -0.02098416 & 0.02213454 & 0.0333094 & 0.04930259 \\
\hline Hyp & -0.05277788 & -0.0331125 & -0.01935216 & 0.0190764 & 0.03103078 & 0.04806589 \\
\hline NIG & -0.06137297 & -0.03517588 & -0.01913473 & 0.01871592 & 0.03152274 & 0.05210736 \\
\hline $\mathrm{VG}$ & -0.05134968 & -0.0326092 & -0.01927233 & 0.01920303 & 0.03109179 & 0.04776555 \\
\hline GHSt & -0.08283004 & -0.03608319 & -0.01850445 & 0.01844769 & 0.03140874 & 0.0574016 \\
\hline Burr & -0.04666 & -0.03138 & -0.02049 & 0.01653 & 0.02609 & 0.0393 \\
\hline JSU & -0.07787022 & -0.038889 & -0.01991437 & 0.01867114 & 0.03335821 & 0.06315589 \\
\hline $\mathrm{HSec}$ & -0.04858 & -0.03105 & -0.01878 & 0.01993 & 0.0322 & 0.04973 \\
\hline Dag & -0.04025 & -0.02661 & -0.0166 & 0.0252 & 0.03801 & 0.05599 \\
\hline GEVD5 & -0.04953552 & -0.031618043 & -0.01854755 & 0.01845054 & 0.030345798 & 0.048066972 \\
\hline GEVD10 & -0.048916708 & -0.031629163 & -0.017691954 & 0.016640345 & 0.029408631 & 0.055409206 \\
\hline GEVD21 & -0.05453539 & -0.029156564 & -0.014162166 & 0.014557746 & 0.027243947 & 0.060748366 \\
\hline GPD85 & -0.052845058 & -0.034189447 & -0.019852259 & 0.018247854 & 0.031892433 & 0.059162774 \\
\hline GPD90 & -0.053026273 & -0.033981172 & -0.019829278 & 0.018642444 & 0.032257528 & 0.052961141 \\
\hline GPD95 & -0.053202975 & -0.033695439 & -0.020104445 & 0.018528649 & 0.032611754 & 0.051527015 \\
\hline
\end{tabular}

Table 8-2: VaR Backtesting for RAFI versus Heavy-Tailed Distributions

\begin{tabular}{|c|c|c|c|c|c|c|c|c|c|c|c|c|c|c|c|c|c|c|}
\hline \multirow[b]{2}{*}{ Distr } & \multicolumn{6}{|c|}{ Number of Violations } & \multicolumn{6}{|c|}{$p$-value of Kupiec Test } & \multicolumn{6}{|c|}{$p$-value of Christoffersen Test } \\
\hline & $0.1 \%$ & $1 \%$ & $5 \%$ & $95 \%$ & 99\% & $99.9 \%$ & $0.1 \%$ & $1 \%$ & $5 \%$ & $95 \%$ & $99 \%$ & $99.9 \%$ & $0.1 \%$ & $1 \%$ & $5 \%$ & $95 \%$ & $99 \%$ & $99.9 \%$ \\
\hline Normal & 18 & 55 & 135 & 100 & 42 & 15 & $<0.001$ & $<0.001$ & 0.3672 & 0.0174 & 0.0019 & $<0.001$ & $<0.001$ & $<0.001$ & 0.0182 & 0.0017 & $<0.001$ & $<0.001$ \\
\hline$t$ & 4 & 29 & 115 & 76 & 24 & 4 & 0.3834 & 0.4342 & 0.3502 & $<0.001$ & 0.8381 & 0.3834 & 0.6796 & 0.5241 & 0.0456 & $<0.001$ & 0.0666 & 0.6796 \\
\hline Hyp & 3 & 25 & 132 & 119 & 35 & 4 & 0.7596 & 0.9984 & 0.5273 & 0.5759 & 0.0583 & 0.3834 & 0.9508 & 0.7768 & 0.0351 & $<0.001$ & 0.0074 & 0.6796 \\
\hline NIG & 1 & 19 & 135 & 123 & 32 & 2 & 0.2795 & 0.2072 & 0.3672 & 0.8504 & 0.1781 & 0.7425 & 0.5570 & 0.3903 & 0.0182 & 0.0015 & 0.0110 & 0.9460 \\
\hline VG & 4 & 26 & 134 & 118 & 34 & 4 & 0.3834 & 0.8433 & 0.4167 & 139 & 67 & 0.3834 & 0.6796 & 0.7462 & & 01 & 0.0088 & 0.6796 \\
\hline $\mathrm{GHS} t$ & 0 & 18 & 141 & 127 & 32 & 0 & 0.0253 & 0.1381 & 0.1512 & 0.8584 & 0.1781 & 0.0253 & 0.0819 & 0.2922 & 0.0093 & 0.0030 & 0.0110 & 0.0819 \\
\hline Burr & 4 & 31 & 120 & 158 & 50 & 14 & 0.3834 & 0.2459 & 0.6410 & 0.0036 & $<0.001$ & $<0.001$ & 0.6796 & 0.3456 & 0.0482 & $<0.001$ & $<0.001$ & $<0.001$ \\
\hline JSU & 0 & 13 & 127 & 124 & 24 & 0 & 0.0253 & 0.0079 & 0.8584 & 0.9232 & 0.8381 & 0.0253 & 0.0819 & 0.0273 & 0.0525 & 0.0018 & 0.0666 & 0.0819 \\
\hline HSec & 4 & 32 & 138 & 110 & 27 & 3 & 0.3834 & 0.1781 & 0.2422 & 0.1590 & 0.6930 & 0.7596 & 0.6796 & 0.2667 & 0.0202 & 0.0053 & 0.0970 & 0.9508 \\
\hline Dag & 11 & 59 & 181 & 53 & 14 & 0 & $<0.001$ & $<0.001$ & $<0.001$ & $<0.001$ & 0.0158 & 0.0253 & $<0.001$ & $<0.001$ & $<0.001$ & $<0.001$ & 0.0101 & 0.0819 \\
\hline GEVD5 & 4 & 30 & 140 & 127 & 37 & 4 & 0.3834 & 0.3309 & 0.1780 & 0.8584 & 0.0245 & 0.3834 & 0.6796 & 0.4330 & 0.0092 & 0.0030 & 0.0047 & 0.6796 \\
\hline GEVD10 & 4 & 30 & 154 & 156 & 42 & 0 & 0.3834 & 0.3309 & 0.0102 & 0.0062 & 0.0019 & 0.0253 & 0.6796 & 0.4330 & $<0.001$ & $<0.001$ & $<0.001$ & 0.0819 \\
\hline GEVD21 & 3 & 48 & 238 & 206 & 47 & 0 & 0.7596 & $<0.001$ & $<0.001$ & $<0.001$ & $<0.001$ & 0.0253 & 0.9508 & $<0.001$ & $<0.001$ & $<0.001$ & $<0.001$ & 0.0819 \\
\hline GPD85 & 3 & 21 & 128 & 131 & 29 & 0 & 0.7596 & 0.4072 & 0.7874 & 0.5879 & 0.4342 & 0.0253 & 0.9508 & 0.5937 & 0.0248 & 0.0018 & 0.0996 & 0.0819 \\
\hline GPD90 & 3 & 22 & 128 & 124 & 27 & 2 & 0.7596 & 0.5369 & 0.7874 & 0.9232 & 0.6930 & 0.7425 & 0.9508 & 0.6798 & 0.0248 & 0.0018 & 0.0970 & 0.9460 \\
\hline GPD95 & 3 & 22 & 124 & 126 & 27 & 2 & 0.7596 & 0.5369 & 0.9232 & 0.9306 & 0.6930 & 0.7425 & 0.9508 & 0.6798 & 0.0368 & 0.0026 & 0.0970 & 0.9460 \\
\hline
\end{tabular}


Table 9-1: VaR Estimates for FINDI using Heavy-Tailed Distributions

\begin{tabular}{|c|c|c|c|c|c|c|}
\hline \multirow[b]{2}{*}{ Distr } & \multicolumn{6}{|c|}{ VaR Estimates } \\
\hline & $0.1 \%$ & $1 \%$ & $5 \%$ & $95 \%$ & $99 \%$ & $99.9 \%$ \\
\hline Historical & -0.04254271 & -0.03060448 & -0.01688861 & 0.01650247 & 0.03080411 & 0.04290212 \\
\hline Normal & -0.03248829 & -0.02428472 & -0.01696597 & 0.01836311 & 0.02568186 & 0.03388543 \\
\hline$t$ & -0.04295248 & -0.02865968 & -0.01865087 & 0.02004801 & 0.03005682 & 0.04434962 \\
\hline Hyp & -0.04819379 & -0.03013057 & -0.01748241 & 0.01694143 & 0.02706588 & 0.04145187 \\
\hline NIG & -0.05505486 & -0.03165666 & -0.01721803 & 0.0167729 & 0.02765248 & 0.04489185 \\
\hline VG & -0.0473395 & -0.02995412 & -0.01758038 & 0.0169437 & 0.02684473 & 0.04075536 \\
\hline $\mathrm{GHS} t$ & -0.07348652 & -0.03225621 & -0.01652153 & 0.01669449 & 0.02768841 & 0.04893703 \\
\hline Burr & -0.04153 & -0.02792 & -0.01822 & 0.01478 & 0.02331 & 0.03512 \\
\hline JSU & -0.05873317 & -0.0314563 & -0.01692576 & 0.01646147 & 0.02730146 & 0.04722285 \\
\hline $\mathrm{HSec}$ & -0.04344 & -0.0277 & -0.01668 & 0.01808 & 0.0291 & 0.04484 \\
\hline Dag & -0.04145 & -0.02744 & -0.01688 & 0.01625 & 0.02464 & 0.03687 \\
\hline GEVD5 & -0.044887841 & -0.027965703 & -0.016180517 & 0.01652948 & 0.027254575 & 0.043443046 \\
\hline GEVD10 & -0.043058244 & -0.027467348 & -0.015452632 & 0.014786947 & 0.0265889 & 0.052470224 \\
\hline GEVD21 & -0.045995645 & -0.025410914 & -0.013046802 & 0.013612306 & 0.024529679 & 0.054050499 \\
\hline GPD85 & -0.047990551 & -0.030130332 & -0.017457649 & 0.016509658 & 0.02887113 & 0.05190397 \\
\hline GPD90 & -0.048644606 & -0.030013653 & -0.017323262 & 0.016543844 & 0.028875464 & 0.051455572 \\
\hline GPD95 & -0.048533292 & -0.030380116 & -0.016885171 & 0.016499195 & 0.029591885 & 0.048028699 \\
\hline
\end{tabular}

Table 9-2: VaR Backtesting for FINDI versus Heavy-Tailed Distributions

\begin{tabular}{|c|c|c|c|c|c|c|c|c|c|c|c|c|c|c|c|c|c|c|}
\hline \multirow[b]{2}{*}{ Distr } & \multicolumn{6}{|c|}{ Number of Violations } & \multicolumn{6}{|c|}{$p$-value of Kupiec Test } & \multicolumn{6}{|c|}{$p$-value of Christoffersen Test } \\
\hline & $0.1 \%$ & $1 \%$ & $5 \%$ & $95 \%$ & $99 \%$ & $99.9 \%$ & $0.1 \%$ & $1 \%$ & $5 \%$ & $95 \%$ & $99 \%$ & $99.9 \%$ & $0.1 \%$ & $1 \%$ & $5 \%$ & $95 \%$ & $99 \%$ & $99.9 \%$ \\
\hline Normal & 19 & 53 & 125 & 98 & 44 & 16 & $<0.001$ & $<0.001$ & 0.9963 & 0.0100 & $<0.001$ & $<0.001$ & $<0.001$ & $<0.001$ & 0.3420 & $<0.001$ & $<0.001$ & $<0.001$ \\
\hline$t$ & 3 & 33 & 98 & 81 & 27 & 3 & 0.7596 & 0.1258 & 0.0100 & $<0.001$ & 0.6930 & 0.7596 & 0.9508 & 0.0651 & 0.0054 & $<0.001$ & 0.0970 & 0.9508 \\
\hline Hyp & 2 & 26 & 118 & 115 & 40 & 3 & 0.7425 & 0.8433 & 5139 & 0.3502 & 0.0056 & 0.7596 & 0.9460 & 0.0897 & 0.1626 & 0.0023 & $<0.001$ & 0.9508 \\
\hline NIG & 2 & 20 & 123 & 119 & 39 & 2 & 0.7425 & 0.2968 & 0.8504 & 0.5759 & 0.0094 & 0.7425 & 0.9460 & 0.0196 & 0.2922 & 0.001 & 0.001 & 0.9460 \\
\hline VG & 2 & 28 & 117 & 115 & 40 & 4 & 0.7425 & 0.5555 & 0.4555 & 0.3502 & 0.0056 & 0.3834 & 0.9460 & 0.1004 & 0.1398 & 0.0023 & $<0.001$ & 0.6796 \\
\hline GHSt & 0 & 20 & 130 & 122 & 38 & 2 & 0.0253 & 0.2968 & 0.6517 & 0.7788 & 0.0153 & 0.7425 & 0.0819 & 0.0196 & 0.0302 & 0.0012 & $<0.001$ & 0.9460 \\
\hline Burr & 3 & 35 & 105 & 154 & 52 & 13 & 0.7596 & 0.0583 & 0.0588 & 0.0102 & $<0.001$ & $<0.001$ & 0.9508 & 0.0423 & 0.0217 & $<0.001$ & $<0.001$ & $<0.001$ \\
\hline JSU & 2 & 21 & 125 & 127 & 40 & 2 & 0.7425 & 0.4072 & 0.9963 & 0.8584 & 0.0056 & 0.7425 & 0.9460 & 0.0290 & 0.3420 & $<0.001$ & $<0.001$ & 0.9460 \\
\hline $\mathrm{HSec}$ & 3 & 35 & 127 & 102 & 33 & 2 & 0.7596 & 0.0583 & 0.8584 & 0.0291 & 0.1258 & 0.7425 & 0.9508 & 0.0423 & 0.1130 & 0.0012 & 0.0100 & 0.9460 \\
\hline Dag & 4 & 36 & 126 & 130 & 45 & 7 & 0.3834 & 0.0382 & 0.9306 & 0.6517 & $<0.001$ & 0.0199 & 0.6796 & 0.0324 & 0.2049 & $<0.001$ & $<0.001$ & 0.0030 \\
\hline GEVD5 & 2 & 34 & 136 & 125 & 40 & 3 & 0.7425 & 0.0867 & 0.3216 & 0.9963 & 0.0056 & 0.7596 & 0.9460 & 0.0534 & 0.0419 & $<0.001$ & $<0.001$ & 0.9508 \\
\hline GEVD10 & 3 & 36 & 158 & 154 & 41 & 2 & 0.7596 & 0.0382 & 0.0036 & 0.0102 & 0.0033 & 0.7425 & 0.9508 & 0.0324 & $<0.001$ & $<0.001$ & $<0.001$ & 0.9460 \\
\hline GEVD21 & 2 & 46 & 218 & 181 & 45 & 2 & 0.7425 & $<0.001$ & $<0.001$ & $<0.001$ & $<0.001$ & 0.7425 & 0.9460 & $<0.001$ & $<0.001$ & $<0.001$ & $<0.001$ & 0.9460 \\
\hline GPD85 & 2 & 26 & 118 & 125 & 33 & 2 & 0.7425 & 0.8433 & 0.5139 & 0.9963 & 0.1258 & 0.7425 & 0.9460 & 0.0897 & 0.1626 & $<0.001$ & 0.0100 & 0.9460 \\
\hline GPD90 & 2 & 27 & 120 & 124 & 33 & 2 & 0.7425 & 0.6930 & 0.6410 & 0.9232 & 0.1258 & 0.7425 & 0.9460 & 0.0970 & 0.2126 & $<0.001$ & 0.0100 & 0.9460 \\
\hline GPD95 & 2 & 26 & 126 & 126 & 31 & 2 & 0.7425 & 0.8433 & 0.9306 & 0.9306 & 0.2459 & 0.7425 & 0.9460 & 0.0897 & 0.2049 & $<0.001$ & 0.0116 & 0.9460 \\
\hline
\end{tabular}


Table 10-1: VaR Estimates for CALSI using Heavy-Tailed Distributions

\begin{tabular}{|c|c|c|c|c|c|c|}
\hline \multirow[b]{2}{*}{ Distr } & \multicolumn{6}{|c|}{ VaR Estimates } \\
\hline & $0.1 \%$ & $1 \%$ & $5 \%$ & $95 \%$ & $99 \%$ & $99.9 \%$ \\
\hline Historical & -0.06295644 & -0.03338476 & -0.01954707 & 0.01828953 & 0.03272231 & 0.05643041 \\
\hline Normal & -0.03766442 & -0.02820333 & -0.01976269 & 0.02098198 & 0.02942262 & 0.03888371 \\
\hline$t$ & -0.05011895 & -0.03339734 & -0.02175973 & 0.02297902 & 0.03461663 & 0.05133825 \\
\hline Hyp & -0.05405648 & -0.03403098 & -0.0199731 & 0.01951675 & 0.03134565 & 0.04818933 \\
\hline NIG & -0.06162635 & -0.03578807 & -0.01976096 & 0.01927992 & 0.03200351 & 0.05211939 \\
\hline VG & -0.05260507 & -0.03358922 & -0.0199824 & 0.0196674 & 0.03139124 & 0.04776451 \\
\hline GHSt & -0.07944913 & -0.0362653 & -0.01900618 & 0.019055 & 0.03190365 & 0.05683948 \\
\hline Burr & -0.04587 & -0.03069 & -0.01986 & 0.0178 & 0.02792 & 0.04201 \\
\hline JSU & -0.06318285 & -0.03500696 & -0.01943994 & 0.01840708 & 0.03008193 & 0.05070773 \\
\hline $\mathrm{HSec}$ & -0.05031 & -0.03215 & -0.01944 & 0.02066 & 0.03337 & 0.05153 \\
\hline Dag & -0.04134 & -0.02924 & -0.01987 & 0.02769 & 0.04355 & 0.06587 \\
\hline GEVD5 & -0.051128188 & -0.032111587 & -0.018821711 & 0.018917662 & 0.031191398 & 0.049754191 \\
\hline GEVD10 & -0.050234251 & -0.031798967 & -0.017941304 & 0.017002319 & 0.029950466 & 0.055535787 \\
\hline GEVD21 & -0.05399263 & -0.029408182 & -0.014814386 & 0.014824084 & 0.027407832 & 0.071281936 \\
\hline GPD85 & -0.057602946 & -0.034899852 & -0.01988702 & 0.018717626 & 0.032991516 & 0.061633688 \\
\hline GPD90 & -0.058256603 & -0.034763567 & -0.01980496 & 0.018555241 & 0.032852433 & 0.064932041 \\
\hline GPD95 & -0.057528835 & -0.035294933 & -0.019543134 & 0.018360101 & 0.033841294 & 0.05771447 \\
\hline
\end{tabular}

Table 10-2: VaR Backtesting for CALSI versus Heavy-Tailed Distributions

\begin{tabular}{|c|c|c|c|c|c|c|c|c|c|c|c|c|c|c|c|c|c|c|}
\hline & \multicolumn{6}{|c|}{ Number of Violations } & \multicolumn{6}{|c|}{$p$-value of Kupiec Test } & \multicolumn{6}{|c|}{$p$-value of Christoffersen Test } \\
\hline Distr & $0.1 \%$ & $1 \%$ & $5 \%$ & $95 \%$ & $99 \%$ & $99.9 \%$ & $0.1 \%$ & $1 \%$ & $5 \%$ & $95 \%$ & $99 \%$ & $99.9 \%$ & $0.1 \%$ & $1 \%$ & $5 \%$ & $95 \%$ & $99 \%$ & $99.9 \%$ \\
\hline Normal & 18 & 54 & 124 & 93 & 37 & 16 & $<0.001$ & $<0.001$ & 0.9232 & 0.0021 & 0.0245 & $<0.001$ & $<0.001$ & $<0.001$ & 0.0368 & $<0.001$ & 0.0047 & $<0.001$ \\
\hline$t$ & 5 & 25 & 103 & 75 & 22 & 4 & 0.1645 & 0.9984 & 0.0372 & $<0.001$ & 0.5369 & 0.3834 & 0.3768 & 0.0792 & 0.0120 & $<0.001$ & 0.0404 & 0.6796 \\
\hline Hyp & 5 & 23 & 122 & 112 & 36 & 9 & 0.1645 & 0.6822 & 0.7788 & 0.2232 & 0.0382 & 0.0015 & 0.3768 & 0.7427 & 0.0650 & $<0.001$ & 0.0060 & $<0.001$ \\
\hline NIG & 3 & 20 & 124 & 112 & 32 & 4 & 0.7596 & 0.2968 & 0.9232 & 0.2232 & 0.1781 & 0.3834 & 0.9508 & 0.4939 & 0.0368 & $<0.001$ & 0.0766 & 0.6796 \\
\hline VG & 5 & 25 & 122 & 109 & 36 & 10 & 0.1645 & 0.9984 & 0.7788 & 0.1325 & 0.0382 & $<0.001$ & 0.3768 & 0.0792 & 0.0650 & $<0.001$ & 0.0060 & $<0.001$ \\
\hline GHSt & 0 & 20 & 132 & 113 & 32 & 3 & 0.0253 & 0.2968 & 0.5273 & 0.2614 & 0.1781 & 0.7596 & 0.0819 & 0.4939 & 0.0149 & 0.0013 & 0.0766 & 0.9508 \\
\hline Burr & 6 & 36 & 123 & 131 & 44 & 15 & 0.0611 & 0.0382 & 0.8504 & 0.5879 & $<0.001$ & $<0.001$ & 0.1706 & 0.0060 & 0.0743 & $<0.001$ & $<0.001$ & $<0.001$ \\
\hline JSU & 3 & 21 & 126 & 124 & 36 & 5 & 0.7596 & 0.4072 & 0.9306 & 0.9232 & 0.0382 & 0.1645 & 0.9508 & 0.5937 & 0.0472 & $<0.001$ & 0.0060 & 0.3768 \\
\hline $\mathrm{HSec}$ & 5 & 30 & 126 & 96 & 24 & 4 & 0.1645 & 0.3309 & 0.9306 & 0.0055 & 0.8381 & 0.3834 & 0.3768 & 0.0118 & 0.0472 & $<0.001$ & 0.0666 & 0.6796 \\
\hline Dag & 11 & 48 & 123 & 46 & 13 & 0 & $<0.001$ & $<0.001$ & 0.8504 & $<0.001$ & 0.0079 & 0.0253 & $<0.001$ & $<0.001$ & 0.0743 & $<0.001$ & $<0.001$ & 0.0819 \\
\hline GEVD5 & 5 & 30 & 133 & 115 & 36 & 6 & 0.1645 & 0.3309 & 0.4701 & 0.3502 & 0.0382 & 0.0611 & 0.3768 & 0.0118 & 0.0161 & 0.0023 & 0.0060 & 0.0056 \\
\hline GEVD10 & 5 & 30 & 151 & 151 & 36 & 3 & 0.1645 & 0.3309 & 0.0209 & 0.0209 & 0.0382 & 0.7596 & 0.3768 & 0.0118 & $<0.001$ & $<0.001$ & 0.0060 & 0.9508 \\
\hline GEVD21 & 5 & 45 & 227 & 207 & 47 & 0 & 0.1645 & $<0.001$ & $<0.001$ & $<0.001$ & $<0.001$ & 0.0253 & 0.3768 & $<0.001$ & $<0.001$ & $<0.001$ & $<0.001$ & 0.0819 \\
\hline GPD85 & 4 & 21 & 123 & 117 & 25 & 1 & 0.3834 & 0.4072 & 0.8504 & 0.4555 & 0.9984 & 0.2795 & 0.6796 & 0.5937 & 0.0743 & 0.0012 & 0.0792 & 0.5570 \\
\hline GPD90 & 4 & 21 & 124 & 122 & 25 & 0 & 0.3834 & 0.4072 & 0.9232 & 0.7788 & 0.9984 & 0.0253 & 0.6796 & 0.5937 & 0.0368 & 0.0012 & 0.0792 & 0.0819 \\
\hline GPD95 & 4 & 21 & 126 & 124 & 23 & 3 & 0.3834 & 0.4072 & 0.9306 & 0.9232 & 0.6822 & 0.7596 & 0.6796 & 0.5937 & 0.0472 & $<0.001$ & 0.0533 & 0.9508 \\
\hline
\end{tabular}


Table 11-1: VaR Estimates for SAVI using Heavy-Tailed Distributions

\begin{tabular}{|c|c|c|c|c|c|c|}
\hline \multirow[b]{2}{*}{ Distr } & \multicolumn{6}{|c|}{ VaR Estimates } \\
\hline & $0.1 \%$ & $1 \%$ & $5 \%$ & $95 \%$ & $99 \%$ & $99.9 \%$ \\
\hline Historical & -0.1106904 & -0.06543635 & -0.03962137 & 0.04613077 & 0.09104422 & 0.1523955 \\
\hline Normal & -0.08946097 & -0.06735913 & -0.04764112 & 0.04754159 & 0.0672596 & 0.08936144 \\
\hline$t$ & -0.119959 & -0.0800319 & -0.05250346 & 0.05240393 & 0.07993237 & 0.1198594 \\
\hline Нyp & -0.106403 & -0.06881783 & -0.04245153 & 0.04829278 & 0.08238721 & 0.1310872 \\
\hline NIG & -0.1119719 & -0.0686886 & -0.0414328 & 0.04837206 & 0.08962371 & 0.1574404 \\
\hline VG & -0.1213116 & -0.07427668 & -0.04201986 & 0.04751201 & 0.08281545 & 0.1343157 \\
\hline GHSt & -0.1129284 & -0.06669665 & -0.04097826 & 0.04628706 & 0.09222452 & 0.2244312 \\
\hline Burr & -0.12347 & -0.08287 & -0.05391 & 0.04533 & 0.07136 & 0.10745 \\
\hline JSU & -0.1135567 & -0.06664149 & -0.04016629 & 0.04563555 & 0.08120824 & 0.1458117 \\
\hline $\mathrm{HSec}$ & -0.119 & -0.07657 & -0.04688 & 0.04679 & 0.07647 & 0.1189 \\
\hline Dag & -0.06428 & -0.05428 & -0.04341 & 0.0854 & 0.15564 & 0.28008 \\
\hline GEVD5 & -0.111054748 & -0.06832769 & -0.041405138 & 0.045719649 & 0.088577905 & 0.172265208 \\
\hline GEVD10 & -0.120882978 & -0.066833803 & -0.038878977 & 0.043327121 & 0.08455424 & 0.164541885 \\
\hline GEVD21 & -0.124127444 & -0.066318321 & -0.037322173 & 0.043257233 & 0.08308032 & 0.160459987 \\
\hline GPD85 & -0.115591867 & -0.067836273 & -0.040858235 & 0.048397602 & 0.088571139 & 0.158081653 \\
\hline GPD90 & -0.122392068 & -0.066121504 & -0.040095258 & 0.047994349 & 0.088140724 & 0.162980192 \\
\hline GPD95 & -0.124284618 & -0.06612048 & -0.039629372 & 0.046146934 & 0.090744711 & 0.15408346 \\
\hline
\end{tabular}

Table 11-2: VaR Backtesting for SAVI versus Heavy-Tailed Distributions

\begin{tabular}{|c|c|c|c|c|c|c|c|c|c|c|c|c|c|c|c|c|c|c|}
\hline & \multicolumn{6}{|c|}{ Number of Violations } & \multicolumn{6}{|c|}{$p$-value of Kupiec Test } & \multicolumn{6}{|c|}{$p$-value of Christoffersen Test } \\
\hline Distr & $0.1 \%$ & $1 \%$ & $5 \%$ & $95 \%$ & $99 \%$ & $99.9 \%$ & $0.1 \%$ & $1 \%$ & $5 \%$ & $95 \%$ & $99 \%$ & $99.9 \%$ & $0.1 \%$ & $1 \%$ & $5 \%$ & $95 \%$ & $99 \%$ & $99.9 \%$ \\
\hline Normal & 4 & 15 & 50 & 80 & 42 & 19 & 0.1382 & 0.5874 & $<0.001$ & 0.5055 & $<0.001$ & $<0.001$ & 0.3302 & 0.7563 & $<0.001$ & 0.0381 & $<0.001$ & $<0.001$ \\
\hline$t$ & 1 & 8 & 40 & 67 & 25 & 5 & 0.5514 & 0.0128 & $<0.001$ & 0.0294 & 0.0762 & 0.0423 & 0.8370 & 0.0434 & $<0.001$ & 0.0055 & 0.1404 & 0.1255 \\
\hline Hyp & 3 & 14 & 72 & 80 & 22 & 3 & 0.3771 & 0.4243 & 0.1127 & 0.5055 & 0.2637 & 0.3771 & 0.6735 & 0.6478 & 0.2841 & 0.0381 & 0.3020 & 0.6735 \\
\hline NIG & 2 & 14 & 76 & 80 & 18 & 1 & 0.8345 & 0.4243 & 0.2617 & 0.5055 & 0.8455 & 0.5514 & 0.9761 & 0.6478 & 0.5003 & 0.0381 & 0.8109 & 0.8370 \\
\hline VG & 1 & 10 & 74 & 80 & 21 & 3 & 0.5514 & 0.0586 & 0.1759 & 0.5055 & 0.3721 & 0.3771 & 0.8370 & 0.1578 & 0.3602 & 0.0381 & 0.3527 & 0.6735 \\
\hline GHSt & 2 & 16 & 77 & 86 & 17 & 0 & 0.8345 & 0.7704 & 0.3137 & 0.9956 & 0.9632 & 0.0636 & 0.9761 & 0.8245 & 0.5751 & 0.0428 & 0.8428 & 0.1791 \\
\hline Burr & 1 & 8 & 35 & 95 & 36 & 12 & 0.5514 & 0.0128 & $<0.001$ & 0.3243 & $<0.001$ & $<0.001$ & 0.8370 & 0.0436 & $<0.001$ & 0.0386 & $<0.001$ & $<0.001$ \\
\hline JSU & 2 & 16 & 82 & 93 & 23 & 3 & 0.8345 & 0.7704 & 0.6597 & 0.4410 & 0.1805 & 0.3771 & 0.9761 & 0.8245 & 0.7781 & 0.0349 & 0.2456 & 0.6735 \\
\hline $\mathrm{HSec}$ & 1 & 9 & 52 & 83 & 30 & 6 & 0.5514 & 0.0289 & $<0.001$ & 0.7427 & 0.0050 & 0.0111 & 0.8370 & 0.0878 & $<0.001$ & 0.0252 & 0.0162 & 0.0390 \\
\hline Dag & 19 & 35 & 68 & 20 & 1 & 0 & $<0.001$ & $<0.001$ & 0.0395 & $<0.001$ & $<0.001$ & 0.0636 & $<0.001$ & $<0.001$ & 0.1180 & $<0.001$ & $<0.001$ & 0.1791 \\
\hline GEVD5 & 2 & 14 & 76 & 93 & 19 & 1 & 0.8345 & 0.4243 & 0.2617 & 0.4410 & 0.6661 & 0.5514 & 0.9761 & 0.6478 & 0.5003 & 0.0349 & 0.7367 & 0.8370 \\
\hline GEVD10 & 1 & 16 & 93 & 107 & 20 & 1 & 0.5514 & 0.7704 & 0.4410 & 0.0246 & 0.5067 & 0.5514 & 0.8370 & 0.8245 & 0.5046 & 0.0021 & 0.6338 & 0.8370 \\
\hline GEVD21 & 1 & 16 & 104 & 108 & 21 & 1 & 0.5514 & 0.7704 & 0.0528 & 0.0187 & 0.3721 & 0.5514 & 0.8370 & 0.8245 & 0.1468 & 0.0020 & 0.3527 & 0.8370 \\
\hline GPD85 & 1 & 14 & 80 & 80 & 19 & 1 & 0.5514 & 0.4243 & 0.5055 & 0.5055 & 0.6661 & 0.5514 & 0.8370 & 0.6478 & 0.6438 & 0.0381 & 0.7367 & 0.8370 \\
\hline GPD90 & 1 & 16 & 83 & 80 & 19 & 1 & 0.5514 & 0.7704 & 0.7427 & 0.5055 & 0.6661 & 0.5514 & 0.8370 & 0.8245 & 0.8353 & 0.0381 & 0.7367 & 0.8370 \\
\hline GPD95 & 1 & 16 & 85 & 86 & 18 & 1 & 0.5514 & 0.7704 & 0.9161 & 0.9956 & 0.8455 & 0.5514 & 0.8370 & 0.8245 & 0.9191 & 0.0428 & 0.8109 & 0.8370 \\
\hline
\end{tabular}




\section{CONCLUSIONS}

In this research, we have made comprehensive examinations in the performances of various heavy-tailed distributions when fitted to eight major JSE indices. The distributions studied include Hyp, NIG, VG, GHSt, Burr, JSU, HSec, Dag, GEVD, and GPD, and are contrasted against the normal distribution, the standard Student's $t$-distribution in the context of goodness-of-fit and the estimation of VaR. Moreover, VaR backtesting procedures provided a uniform measure among these models, which were employed for cross-comparisons between their corresponding relative model performances for extreme tail depictions.

Contrary to prior findings, we show that EVT does not always produce the best model fit for all indices, and similarly for the GHDs. Rather, as partially hinted by Vee et al. (2012), no one best model exist for all financial indices. Although a suitable model may often be identified (i.e., not rejected for any level of VaR by the Kupiec LR test) for a particular return series, it cannot be deemed the optimal distribution for all VaR levels, as shown in our study. Our results suggest that the inconsistency and variation of a best model selection does not only occur across indices, but also across different VaR levels, and dissimilarities also exist between both short and long positions of trade. The demonstration of inconsistencies in the preferred model across different VaR levels is particularly striking. Such a finding further contributes to the contradiction of believe in the existence of a best model to capture all features within a financial returns distribution. Hence, our findings motivate for investigation and possible implementation of stepwise function, mixture of distributions, or model-switching procedures that may better capture the behaviors of financial returns. These may be further explored in prospective further research.

\section{ACKNOWLEDGEMENTS}

The authors would like to thank Mr. Jahvaid Hammujuddy for reading a draft of this article and providing some valuable suggestions.

\section{AUTHOR INFORMATION}

Chun-Sung Huang is a lecturer in Finance and an associate of the African Collaboration for Quantitative Finance and Risk Research (ACQuFRR) at the University of Cape Town. His research interests lie in volatility modeling and forecasting, Value-at-Risk (VaR) modeling, and derivatives pricing in incomplete markets. E-mail: chun-sung.huang@uct.ac.za (Corresponding author)

Chun-Kai Huang is a lecturer in Statistics at the University of Cape Town. He is a member of the South African Statistical Association and a fellow of the Cambridge Commonwealth Society. His research interests lie in probabilistic exchangeability, curve and distribution fitting, moment problems, extreme value theory, and statistical applications to financial data. E-mail: plutokaijuu@gmail.com

Knowledge Chinhamu is a lecturer in Statistics at the University of KwaZulu-Natal and a member of the South African Statistical Association. His research interests lie in financial time series and econometric modeling. E-mail: chinhamu@ukzn.ac.za

\section{REFERENCES}

1. Aas, K., \& Haff, D. H. (2006). The generalized hyperbolic skew Student's t-distribution. Journal of Financial Econometrics, 4(2), 275-309.

2. Abramowitz, M., \& Stegun, A. (1972). Handbook of Mathematical functions with formulas, graphs, and mathematical tables (10th Printing). National Bureau of Standards Applied Mathematics Series 55.

3. Anderson, T. W., \& Darling, D. A. (1952). Asymptotic theory of certain "goodness-of-fit" criteria based on stochastic processes. Annals of Mathematical Statistics, 23, 193-212.

4. Andersson, J. (2001). On the normal inverse Gaussian stochastic volatility model. Journal of Business and Economic Statistics, 19, 44-54.

5. Azzalini, A., \& Capitanio, A. (2003). Distributions generated by perturbation of symmetry with emphasis on a multivariate skew t distribution. Journal of the Royal Statistical Society B, 65(2), 367-389.

6. Bali, T. G. (2003). An extreme value approach to estimating volatility and value at risk. The Journal of Business, 76(1), 83-108.

7. Balkema, A., \& de Haan, L. (1974). Residual life time at great age. Annals of Probability, 2, 792-804. 
8. Banerjee, A., Dolado, J. J., Galbraith, J. W., \& Hendry, D. F. (1993). Cointegration, error correction, and the econometric analysis of non-stationary data. Oxford: Oxford University Press.

9. Barndorff-Nielsen, O. E. (1977). Exponential decreasing distributions of the logarithm of particle size. Proceedings of the Royal Society London A, 353, 401-419.

10. Baten, W. D. (1934). The probability law for the sum of $\mathrm{n}$ independent variables, each subject to the law $(1 /(2 \mathrm{~h})) \operatorname{sech}(\pi \mathrm{x} /(2 \mathrm{~h}))$. Bulletin of the American Mathematical Society, 40(4), 284-290.

11. Burr, I. W. (1942). Cumulative frequency functions. Annals of Mathematical Statistics, 13, $215-232$.

12. Castagliola, P. (1998). Approximation of the normal sample median distribution using symmetrical Johnson SU distributions: application to quality control. Communications in Statistics - Simulation and Computation, 27(2), 289-301.

13. Christoffersen, P. F. (1998). Evaluating interval forecasts. International Economic Review, 39(4), 841-862.

14. Coles, S. (2001). An introduction to statistical modeling of extreme values. Springer Series in Statistics. London: Springer-Verlag.

15. da Silva, A. L. C., \& de Melo Mendes, B. V. (2003). Value-at-risk and extreme returns in Asian stock markets. International Journal of Business, 8(1), 17-40.

16. Dagum, C. (1975). A model of income distribution and the conditions of existence of moments of finite order. Bulletin of the International Statistical Institute 46 (Proceeding of the 40th Session of the ISI, Contributed Paper), 199-205.

17. Dagum, C. (1977). A new model of personal income distribution: Specification and estimation. Economie Appliquée, 30, 413-437.

18. Diebold, F. X., Schuermann, T., \& Stroughair, J. D. (2000). Pitfalls and opportunities in the use of extreme value theory in risk management. Journal of Risk Finance, 1(2), 30-35.

19. Eberlein, E., \& Keller, U. (1995). Hyperbolic distributions in finance. Bernoulli, 1(3), 281-299.

20. Eberlein, E., \& Prause, K. (2002). The generalized hyperbolic model: Financial derivatives and risk measures. Mathematical Finance - Bachelier Congress 2000, 245-267.

21. Embrechts, P. (2000). Extreme value theory: Potential and limitations as an integrated risk management tool. Derivatives Use, Trading \& Regulation, 6, 449-456.

22. Embrechts, P., Resnick, S., \& Samorodnitsky, G. (1999). Extreme value theory as a risk management tool. North American Actuarial Journal, 3, 30-41.

23. Farrel, P. J., \& Stewart, K. R. (2006). Comprehensive study of tests for normality and symmetry: Extending the Spiegelhalter test. Journal of Statistical Computing and Simulation, 76(9), 803-816.

24. Fischer, M. (2006). The skew generalized secant hyperbolic family. Austrian Journal of Statistics, 35(4), 437-443.

25. Fisher, R. A., \& Tippett, L. H. C. (1928). Limiting forms of the frequency distribution of the largest and smallest member of a sample. Proc. Cambridge Phil. Soc., 24, 180-190.

26. Gençay, R., \& Selçuk, F. (2004). Extreme value theory and value-at-risk: Relative performance in emerging markets. International Journal of Forecasting, 20, 287-303.

27. Gilli, M., \& Këllezi, E. (2006). An application of extreme value theory for measuring financial risk. Computational Economics, 27, 207-228.

28. Gnedenko, B. V. (1943). Sur la distribution limite du terme maximum d'une série aléatoire. Annals of Mathematics, 44, 423-453.

29. Harkness, W. L., \& Harkness, M. L. (1968). Generalized hyperbolic secant distributions. Journal of the American Statistical Association, 63, 329-337.

30. Ho, L., Burridge, P., Cadle, J., \& Theobald, M. (2000). Value-at-risk: Applying the extreme value approach to Asian markets in the recent financial turmoil. Pacific-Basin Finance Journal, 8, 249-275.

31. Hosking, J. R. M., \& Wallis, J. R. (1987). Parameter and quantile estimation for the generalized Pareto distribution. Technometrics, 29(3), 339-349.

32. Hu, W., \& Kercheval, A. (2007). Risk management with generalized hyperbolic distributions, in Proceedings of the Fourth IASTED International Conference on Financial Engineering and Applications, 19-24.

33. Huisman, R., Koedijk, K. G., \& Pownall, R. A. J. (1998). VaR-x: fat tails in financial risk management. Journal of Risk, 1(1), 47-61.

34. Johnson, N. L. (1949). Systems of frequency curves generated by methods of translation. Biometrika, 36, 149-176.

35. Jorion, P. (2006). Value at risk: The new benchmark for managing financial risk (3rd ed.). McGraw-Hill.

36. Jones, M. C., \& Faddy, M. J. (2003). A skew extension of the t distribution, with applications. Journal of the Royal Statistical Society B, 65(1), 159-174. 
37. Kolmogorov, A. (1933). Sulla determinazione empirica di una legge di distribuzione. G. Ist. Ital. Attuari, 4, 83-91.

38. Kupiec, P. (1995). Techniques for verifying the accuracy of risk measurement models. Journal of Derivatives, 2, 173-184.

39. Madan, D. B., \& Seneta, E. (1990). The variance gamma model for share market returns. Journal of Business, 63, 511-524.

40. McNeil, A., Frey, R., \& Embrechts, P. (2005). Quantitative risk management: Concepts, techniques, and tools. Princeton Univ. Press.

41. McNeil, A., \& Frey, R. (2000). Estimation of tail-related risk measures for heteroscedastic financial time series: an extreme value approach. Journal of Empirical Finance, 7(3-4), 271-300.

42. Monroy, B. S., Huerta, H. V., \& Arnold, B. (2013). Use of the Dagum distribution for modeling tropospheric ozone levels. Journal of Environmental Statistics, 5(5), 1-11.

43. Palmitesta, P., \& Provasi, C. (2004). GARCH-type models with generalized secant hyperbolic innovations. Studies in Nonlinear Dynamics \& Econometrics, 8(2), Article 7.

44. Pickands, J. (1975). Statistical inference using extreme order statistics. Annals of Statistics, 3, 119-131.

45. Prause, K. (1999). The generalized hyperbolic model: Estimation, financial derivatives and risk measures. (Doctoral Thesis). University of Freiburg.

46. Raubenheimer, H. (2012). Managing portfolio managers: the impacts of market concentration, cross-sectional return dispersion and restrictions on short sales. (Doctoral dissertation). Stellenbosch University. Retrieved Jan. 2014 from http://scholar.sun.ac.za/handle/10019.1/20266

47. Rocco, M. (2014). Extreme value theory in finance: a survey. Journal of Economic Surveys, 28(1), 82-108.

48. Rydberg, T. H. (1999). Generalized hyperbolic diffusions with applications towards finance. Mathematical Finance, 9, 183-201.

49. Shao, Q., Wong, H., Xia, J., \& Ip, W. (2004). Models for extremes using the extended three-parameter Burr XII system with application to flood frequency analysis. Hydrological Sciences Journal, 49(4), 685-702.

50. Simonato, J. (2011). The performance of Johnson distributions for computing value at risk and expected shortfall. The Journal of Derivatives, 19(1), 7-24.

51. Singh, S. K., \& Maddala, G. S. (1976). A function for the size distribution of incomes. Econometrica, 44, 963-970.

52. Smirnov, N. (1948). Table for estimating the goodness of fit of empirical distributions. Annals of Mathematical Statistics, 19, 279-281.

53. Tejeda, H. A., \& Goodwin, B. K. (2008). Modeling crop prices through a Burr distribution and analysis of correlation between crop prices and yields using a copula method, paper presented at The annual meeting of the Agricultural and Applied Economics Association, Orlando, FL. http://purl.umn.edu/6061

54. Tsay, R. S. (2010). Analysis of financial time series (3rd ed.). Wiley \& Sons.

55. Tsay, R. S. (2013). An introduction to analysis of financial data with R. Wiley \& Sons.

56. Vee, D. N. C., Gonpot, P. N., \& Sookia, N. (2012). Assessing the performance of generalized autoregressive conditional heteroskedasticity-based value-at-risk models: a case of frontier markets. Journal of Risk Model Validation, 6(4), 95-111.

57. Venter, J. H., \& de Jongh, P. J. (2002). Risk estimation using the normal inverse Gaussian distribution. Journal of Risk, 4(2), 1-24.

58. Wheeler, R. E. (1980). Quantile estimators of Johnson curve parameters. Biometrika, 67(3), 725-728.

59. The Global Competitiveness Report 2013-2014, World Economic Forum. 